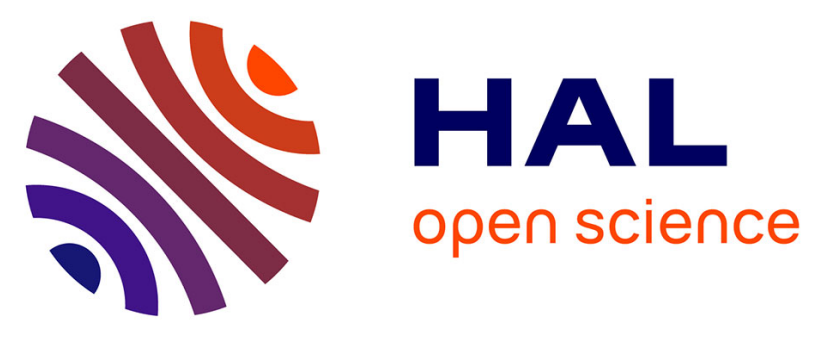

\title{
General treatment of off-specular resonant soft x-ray magnetic scattering using the distorted-wave Born approximation: Numerical algorithm and experimental studies with hybrid chiral domain structures
}

Samuel Flewett, Mackarena Garrido Strelow, Erick Burgos Parra, Yanis Sassi, Fernando Ajejas, N. Reyren, Cyril Léveillé, Nicolas Jaouen

\section{To cite this version:}

Samuel Flewett, Mackarena Garrido Strelow, Erick Burgos Parra, Yanis Sassi, Fernando Ajejas, et al.. General treatment of off-specular resonant soft x-ray magnetic scattering using the distortedwave Born approximation: Numerical algorithm and experimental studies with hybrid chiral domain structures. Physical Review B, 2021, 103 (18), 10.1103/PhysRevB.103.184401 . hal-03318072

\author{
HAL Id: hal-03318072 \\ https://hal.science/hal-03318072
}

Submitted on 9 Aug 2021

HAL is a multi-disciplinary open access archive for the deposit and dissemination of scientific research documents, whether they are published or not. The documents may come from teaching and research institutions in France or abroad, or from public or private research centers.
L'archive ouverte pluridisciplinaire HAL, est destinée au dépôt et à la diffusion de documents scientifiques de niveau recherche, publiés ou non, émanant des établissements d'enseignement et de recherche français ou étrangers, des laboratoires publics ou privés. 


\title{
General treatment of off-specular resonant soft $x$-ray magnetic scattering using the distorted-wave Born approximation: Numerical algorithm and experimental studies with hybrid chiral domain structures
}

\author{
Samuel Flewett* and Mackarena Garrido Strelow \\ Pontificia Universidad Católica de Valparaíso, Avenida Unviersidad 330, Valparaíso, CHILE \\ Erick Burgos Parra \\ Synchrotron SOLEIL, Saint-Aubin, Boite Postale 48, 91192 Gif-sur-Yvette Cedex, FRANCE and \\ Unite Mixte de Physique, CNRS, Thales, Universite Paris-Saclay, 91767 Palaiseau, France \\ Yanis Sassi, Fernando Ajejas, and Nicolas Reyren \\ Unite Mixte de Physique, CNRS, Thales, Universite Paris-Saclay, 91767 Palaiseau, FRANCE \\ Cyril Léveillé and Nicolas Jaouen \\ Synchrotron SOLEIL, Saint-Aubin, Boite Postale 48, 91192 Gif-sur-Yvette Cedex, FRANCE
}

(Dated: January 2021)

\section{CONTENTS}

\section{Abstract}

II. Introduction

II.1. General Background

II.2. Theoretical Background

II.3. Outline of the medium boundary matrix formalism

II.4. Multilayer Sample Treatment

III. Simulations: Procedure and Considerations

III.1. The recipe to calculate the diffraction pattern in reflection conditions

III.2. Introduction to the theme of Roughness in Simulations

III.3. Transmission Geometry

III.4. Treatment of X-ray coherence

IV. Experimental Validation

IV.1. Experimental and Simulation Conditions

IV.2. Interference effects moving off the multilayer Bragg Angle

IV.3. Born Approximation vs. Distorted Wave Born Approximation: Explanation of Dichroism observed with Applied Longitudinal Field in Second Order Scattering Peaks

IV.4. Studying Depth Penetration with Energy Scans at Different Bragg Angles

V. Conclusion

VI. Acknowledgements

References

1

1

1

3

\section{ABSTRACT}

(
tool for the nanometer resolution characterization of magnetic domains in thin film samples [1-8]. However, an important gap in the literature is the lack of a standard protocol for the modelling of resonant scattering, which has limited the degree to which quantitative conclusions may be extracted from experimental data. In this article, we begin by reviewing the theory literature on the topic, and then present our numerical implementation of the framework of Bader and Zak [9-12] applied to a film

We present a numerical algorithm for the simulation of X-ray magnetic scattering (XRMS) in reflection geometry for an arbitrary 3D magnetization distribution over a multilayer sample utilizing the Distorted Wave Born Approximation (DWBA). Our approach combines the medium boundary matrix approach for specular reflection, and the Born approximation typically used for off-specular reflection, combining the two and adding the contribution from transmission through the sample before and after a reflection event. The algorithm is then tested on experimental data from an $\mathrm{Ir}|\mathrm{Co}| \mathrm{Pt}$ multilayer sample with hybrid Néel/Bloch/Néel domain walls at a range of incidence angles and photon energies near the $\mathrm{Co}_{3}$ absorption edge, achieving high levels of agreement components into the algorithm was found to explain the dichroism observed in scattering from Bloch type domain walls, and uncovered the likely importance of diffuse scattering in transmission from the poly-crystalline grain walls along the optical path of the X-rays in the sample - a theme which deserves further investigation.

\section{INTRODUCTION}

\section{II.1. General Background}

Over the past 20 years, the field of resonant elastic soft X-ray scattering has widely expanded into a standard

\footnotetext{
* Institutional: samuel.flewett@pucv.cl
} f .

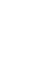


whose magnetization not only varies in the out of plane direction but also in the plane of the sample. Our implementation allows the matrix formalism of Zak et al.[9-12] to be readily applied in 3D over a multilayer with inhomogeneous magnetization by making use of a the Distorted Wave Born approximation (DWBA) in reflection geometry. Here, we calculate the reflection coefficients for each numerical pixel in a "pancake stack" of imperfect reflecting surfaces (corresponding to each interface in a multilayer sample), and propagate the beam through the sample before and after each reflection event in order to account for differences in the absorption and phase shifts due to components of the magnetization parallel to the beam direction.

This research field can be said to have begun with the seminal publication by Dürr et al. in the year 1999 [2], where the characteristic asymmetric scattering pattern was first experimentally demonstrated with soft X-rays for a stripe domain sample. This asymmetry is due to the presence of Néel domain caps on the surfaces of the thin film in between the dominant out of plane domains, and the differing polarization dependent response of the regions magnetized parallel to the scattering plane, versus perpendicular to the scattering plane. These magnetization dependent changes in polarization on reflection are known as the Magnetico Optical Kerr Effect (MOKE). A further development of the work by Dürr, especially in terms of modelling may be found in the work of Beutier et al. [3] In their work, they also started from micromagnetic simulations, which are typically performed using software packages such as OOMMF [13] or MuMax [14]. They start from the atomic level defining the atomic scattering factors for the resonant soft Xray interaction of element $n$ as derived from quantum mechanics [1]

$$
f_{n}=\left(\mathbf{e} \cdot \mathbf{e}^{\prime}\right) f_{c}^{n}+i\left(\mathbf{e} \times \mathbf{e}^{\prime}\right) \cdot \mathbf{M}^{\mathbf{n}} f_{m 1}^{n}+\left(\mathbf{M}^{\mathbf{n}} \cdot \mathbf{e}^{\prime}\right)\left(\mathbf{M}^{\mathbf{n}} \cdot \mathbf{e}\right) f_{m 2}^{n}
$$

where $\mathbf{e}$ is the incoming electric field vector, $\mathbf{e}^{\prime}$ the rotated outgoing electric field vector, $\mathbf{M}^{\mathbf{n}}$ the sample magnetisation vector normalized to unity, $f_{c}^{n}, f_{m 1}^{n}$ and $f_{m 2}^{n}$ the charge, circular dichroic and linear dichroic resonant scattering factors respectively. For the 3d transition metals which will be dealt with in this paper, only the factors $f_{c}^{n}$ and $f_{m 1}^{n}$ have appreciable values, however this is not universally the case, and the $f_{m 2}^{n}$ term needs to be taken into account for example in the case of the multiferroics of Ref. [15]. From these values of the atomic scattering factors, The authors of [3] calculate the scattering factor of each micromagnetic simulation cell, by computing the sum of the atomic scattering factors over each cell assuming constant magnetization across each cell. In turn, they sum each of these cell scattering factors over the the entire magnetic stripe domain period. This Born approximation formalism is similar to that used in crystallography, and accurately predicts the assymetric diffraction which is a signature of stripe domain reflectometry with resonantly tuned soft X-rays. More recent work with soft X-rays where analysis has followed a similar line includes that of Fin et al. [16] where linearly polarized light has been used to study buried domain wall structure in stripe domain samples, the work of Chauleau et al. [4] using circularly polarized light studying worm domain samples, and the study of the internal domain wall structure of skyrmion hosting compounds by Legrand et al. [17]. Zhang et al. [18] used XRMS to probe the 3D structure of a skyrmion array, using atomic transition probabilities to calculate the absorption length and thus the relative weighting of the contributions from each layer within the sample. By invoking an exponential attenuation, the authors of Zhang et al. [18] and also Li et al. [19] are in effect employing the idea of a distorted wave Born approximation, an idea key to this present work. The chirality of a skyrmion array has also been characterized using XRMS, using the orientation of the observed assymetric pattern as a probe of whether the domain walls are of Bloch, Néel or some intermediate state [20]. Resonant scattering was also performed on a patterned NiFe (permalloy) sample by Diaz et al. [21], again utilizing the sum of the scattering factors as the tool for modelling the observed scattering behaviour.

This paper deals with the forward problem of simulating an X-ray scattering pattern given an arbitrary 3D magnetization distribution in a multilayer sample, and demonstrates the validity of the formalism with experimental examples. Compared with previously cited references where the Born approximation is used, an important advantage of our method is the ability to include the effect of transmission on the resulting reflection scattering pattern - something especially important where scattering is performed along Bloch walls with an extended line of interaction between the photons and the sample before and after the reflection event. Differential transmission along the Bloch walls will be shown here to result in a further dichroism additional to the classic asymmetry, a feature which is being exploited by Burgos-Parra et al.[22] to study the evolution of both Bloch and Néel domain walls under external field.

The algorithm presented in this manuscript was developed and improved through the task of analysing experimental data, some of which has already been published [23] or will be soon submitted for publication $[22,24]$, and is an extension of previous transmission geometry work [6, 25]. The experimental material included was specifically chosen to test certain hypotheses which arose from the analysis of the data to be published in [22], namely the ability to quantitatively determine the depth of the Bloch part of the domain wall - a feature believed to be related to the value of the Dzyaloshinskii-Moriya interaction (DMI) [17], and qualitatively explored by Zhang et al. [26]. Tests of our simulation code were also found to predict important changes in the observed diffraction moving on and off the multilayer Bragg peaks due to interference effects, the experimental confirmations of which will also be presented here. As far as we are aware, this effect has 
not yet been published, and magnitude of the effect could have important implications for those wishing to obtain quantitative information about the magnetic structures of their samples. We also begin to examine the effect of roughness and poly-crystalline structure both in reflection and transmission, but due to the complexity of this topic, a full treatment will need to be left for a later manuscript. To fulfil the long term aim of directly recovering the $3 \mathrm{D}$ magnetization distributions from a set of experimental scattering data, it will be necessary to first resolve the theme of roughness and diffuse scattering in transmission from poly-crystalline grain walls, and subsequently (or in parallel) develop an iterative scheme for structure refinement.

\section{II.2. Theoretical Background}

When working with soft X-rays in multilayer samples, it is convenient to consider the sample as a stack of (imperfect) reflective surfaces, each with spatially varying reflection and transmission coefficient tensors depending upon both the local magnetism and electron density.

To find the refractive indices from the atomic scattering factors, the optical theorem [27] can be used which relates the atomic scattering factors to the total permittivity tensor $\epsilon$ as follows

$$
\epsilon=1+\frac{4 \pi r_{e}}{k^{2}} \sum_{n} \rho_{n} \mathbf{F}_{\mathbf{n}}
$$

where $k$ is the wavenumber, $r_{e}$ is the classical electron radius, $\rho_{n}$ is the number of atoms of species $n$ per unit volume, and $\mathbf{F}_{\mathbf{n}}$ is the scattering tensor defined for each type of atom, and the sum is over the elemental species present. $\mathbf{F}_{\mathbf{n}}$ can be expressed $[9-12,28]$

$$
\mathbf{F}_{\mathbf{n}}=\left(\begin{array}{ccc}
f_{c}^{n} & -i f_{m 1}^{n} \mathbf{M}_{\mathbf{z}}^{\mathbf{n}} & i f_{m 1}^{n} \mathbf{M}_{\mathbf{y}}^{\mathbf{n}} \\
i f_{m 1}^{n} \mathbf{M}_{\mathbf{z}}^{\mathbf{n}} & f_{c}^{n} & -i f_{m 1}^{n} \mathbf{M}_{\mathbf{x}}^{\mathbf{n}} \\
-i f_{m 1}^{n} \mathbf{M}_{\mathbf{y}}^{\mathbf{n}} & i f_{m 1}^{n} \mathbf{M}_{\mathbf{x}}^{\mathbf{n}} & f_{c}^{n}
\end{array}\right)
$$

where $\mathbf{M}_{\mathbf{i}}^{\mathbf{n}}$ are the distinct components of the magnetization for elemental species $n$. We may now combine equations 2 and 3, introducing the magnetooptical constant for the $n^{\text {th }}$ element $Q_{n}$ defined as

$$
Q_{n}=\frac{-4 \pi r_{e} \rho_{n}}{k^{2}} f_{m 1}^{n}
$$

and the permittivity for the $n^{t h}$ element as

$$
\epsilon_{n}=1+\frac{4 \pi r_{e} \rho_{n}}{k^{2}} f_{c}^{n}
$$

We therefore obtain the permittivity tensor for each element as

$$
\epsilon_{\mathbf{n}}=\epsilon_{n}\left(\begin{array}{ccc}
1 & i Q_{n} \mathbf{M}_{\mathbf{z}}^{\mathbf{n}} & -i Q_{n} \mathbf{M}_{\mathbf{y}}^{\mathbf{n}} \\
-i Q_{n} \mathbf{M}_{\mathbf{z}}^{\mathbf{n}} & 1 & i Q_{n} \mathbf{M}_{\mathbf{x}}^{\mathbf{n}} \\
i Q_{n} \mathbf{M}_{\mathbf{y}}^{\mathbf{n}} & -i Q_{n} \mathbf{M}_{\mathbf{x}}^{\mathbf{n}} & 1
\end{array}\right)
$$

The refractive index for a given element for circularly polarized light can be then be found from the value of $Q_{n}$ to first order as [9-12]

$$
n=n_{0}\left(1 \pm \sigma g Q_{n} / 2\right)
$$

where $\sigma$ is the helicity of the light and takes a value 1 or $1, n_{0}=\sqrt{\epsilon_{n}}$ is the non dichroic refractive index for element $n$, and $g$ is the cosine of the angle between the magnetization vector $\mathbf{M}$ and the Poynting vector of the incident radiation. This formalism to arrive at the magnetically dependent refractive indices is fully discussed in various references including [29, 30].

With expressions for the magnetically dependent refractive indices, one is in a position to develop a formalism based upon the use of generalized Fresnel formulae, as has been done by various authors as listed in Refs. [9-12, 30-34]. Being based upon the calculation of reflection and transmission coefficients at the different interfaces in a thin film sample (for example a cobalt/palladium multilayer), this theoretical formalism has been extensively utilized in studies of the specular reflection as a function of photon energy and angle of incidence. For example, Tonnerre et al.. [35, 36] probed the thickness dependent magnetization profile of a perpendicular exchanged system with nanometer precision in the $\mathrm{z}$ (out of plane) direction, by scanning the photon energy over the absorption edges of the different elements, and also scanning the incidence angle to probe the multilayer structure. Mertins et al. [37] used the same matrix based method for calculating Kerr rotation values of up to 24 degrees on resonance in the soft X-ray regime, and also corroborated their results with experimental synchrotron data. The magnetic proximity effect was investigated in $\mathrm{Fe} \mid \mathrm{Pt}$ bilayers with hard X-rays at the Pt edge by Kuschel et al. [38, 39] also making use of the energy and angle dependent spectral reflectivity differences, where data analysis was performed by code developed by Macke et al. [29, 40]. This same formalism is further generalized from reflective slab to atomic level resolution by Zwiebler et al. [41] in order to probe the depth sensitive electronic profile with atomic resolution.

A formalism was developed by Valencia et al. to include the interfacial roughness into the simulation [42], achieving an important improvement in fitting the observed experimental data. Elzo et al. [30] similarly included roughness effects in the development of their simulation scheme, with the difference that their formalism was developed using a basis of circular polarization and not linear polarization as with other authors and ourselves.

\section{II.3. Outline of the medium boundary matrix formalism}

In this section, we outline the formalism of Zak et al., however readers wishing a more in-depth understanding of the topic are referred to primary sources of Refs. [9$12,30,32-34]$. The general geometry of the scattering 
process is shown in Fig. 1: At the interface, the tangential components of the electric and magnetic fields $E_{x}, E_{y}, H_{x}, H_{y}$, are conserved, and may be expressed together as a set in the matrix

$$
\mathbf{L}=\left(\begin{array}{l}
E_{x} \\
E_{y} \\
H_{x} \\
H_{y}
\end{array}\right)
$$

At the same time, the components of the incoming and outgoing waves may be expressed in terms of their circular helicities or perpendicular $(\sigma)$ and parallel $(\pi)$ components with respect to the scattering plane which in the geometry chosen here is the $y$ - $z$ plane. Most authors have expressed the medium boundary matrix on a basis of linear polarization as we will here, however Elzo et al. [30] used a circular polarization basis. With linear polarization, the electric fields of the incoming and outgoing (reflected) wave are expressed via the $P_{\text {in }}$ matrix as

$$
\mathbf{P}_{\text {in }}=\left(\begin{array}{c}
E_{\sigma}^{i} \\
E_{\pi}^{i} \\
E_{\sigma}^{r} \\
E_{\pi}^{r}
\end{array}\right)
$$

These two matrices are connected by the medium boundary matrix $A$ according to

$$
\mathbf{L}=A \mathbf{P}_{\text {in }}
$$

Using the notation of Qiu et al. [34], where $\mathbf{Q}_{\mathbf{n}}=$ $\left[Q_{n}^{x}, Q_{n}^{y}, Q_{n}^{z}\right]=Q_{n} \mathbf{M}$ and $n_{0}$ is the non-dichroic refractive index, we have the medium boundary matrix defined as

$$
A_{i}=\left(\begin{array}{cccc}
1 & 0 & 1 & 0 \\
\frac{i}{2}\left[-Q_{n}^{y} \tan \theta\left(1+\cos ^{2} \theta\right)+Q_{n}^{z} \sin ^{2} \theta\right] & \cos \theta+i Q_{n}^{x} \sin \theta & \frac{i}{2}\left[Q_{n}^{y} \tan \theta\left(1+\cos ^{2} \theta\right)+Q_{n}^{z} \sin ^{2} \theta\right] & -\cos \theta+i Q_{n}^{x} \sin \theta \\
\frac{i n_{0}}{2}\left(Q_{n}^{y} \sin \theta+Q_{n}^{z} \cos \theta\right) & -n_{0} & \frac{i n_{0}}{2}\left(Q_{n}^{y} \sin \theta-Q_{n}^{z} \cos \theta\right) & -n_{0} \\
n_{0} \cos \theta & \frac{i n_{0}}{2}\left(Q_{n}^{y} \tan \theta+Q_{n}^{z}\right) & -i n_{0} \cos \theta & \left.Q_{n}^{y} \tan \theta-Q_{n}^{z}\right)
\end{array}\right)
$$

With the medium boundary matrix, we are thus in the position to express the radiation amplitudes on both sides of a reflecting matrix as follows: We first apply Eq. 10 for the interface between material 1 and material 2 of which can either be magnetic with values $Q_{n} \neq 0$, or can be non-magnetic where $Q_{n}=0$.

$$
A_{1} \mathbf{P}_{\mathbf{1}}=A_{2} \mathbf{P}_{\mathbf{2}}
$$

From here, we may express $\mathbf{P}_{\mathbf{1}}$ in terms of $\mathbf{P}_{\mathbf{2}}$ as

$$
\mathbf{P}_{\mathbf{1}}=A_{1}^{-1} A_{2} \mathbf{P}_{\mathbf{2}}=M \mathbf{P}_{\mathbf{2}}
$$

The matrix $M$ is a $4 \times 4$ matrix which may be expressed in terms of four $2 \times 2$ matrices

$$
M=\left(\begin{array}{cc}
G & H \\
I & J
\end{array}\right),
$$

where $I$ is not to be confused with the identity matrix, and from which the transmission and reflection coefficients can be calculated according to

$$
T=G^{-1}=\left(\begin{array}{ll}
t_{\sigma \sigma} & t_{\sigma \pi} \\
t_{\pi \sigma} & t_{\pi \pi}
\end{array}\right)
$$

and

$$
R=I G^{-1}=\left(\begin{array}{ll}
r_{\sigma \sigma} & r_{\sigma \pi} \\
r_{\pi \sigma} & r_{\pi \pi}
\end{array}\right)
$$

With the reflection and transmission coefficients on hand for a single interface, it is immediately apparent how one may calculate the resonant scattering from a single interface between two materials, or between a vacuum and a the surface of a single layer magnetic film. We compute the spatial distribution of the magnetization vector $\mathbf{M}$, over the surface of the film, and then calculate the boundary matrices for both materials at each simulation pixel. Diffraction may then be calculated from the reflection coefficients given the polarization of the incident light, and the spatial array of reflection coefficients calculated at each point using the above described procedure (for example from a perpendicular anisotropy stripe domain sample with closure domains where the diffraction will produces the typical asymmetric 1st order peaks for example in Dürr et al. [2]). In cases where the sample at hand is a smooth and optically thick single-layer sample (not a multilayer), no more work is necessary to simulate the scattering from the surface layer. Such was the state of the algorithm used for the modelling of scattering in the work of Pianciola et al. [23]. For multilayer samples, the treatment is more complex and is presented in the subsequent section.

\section{II.4. Multilayer Sample Treatment}

Due to the fact that the pair of medium boundary matrices expresses the electromagnetic field on one side of an interface in terms of the field on the other side of this 


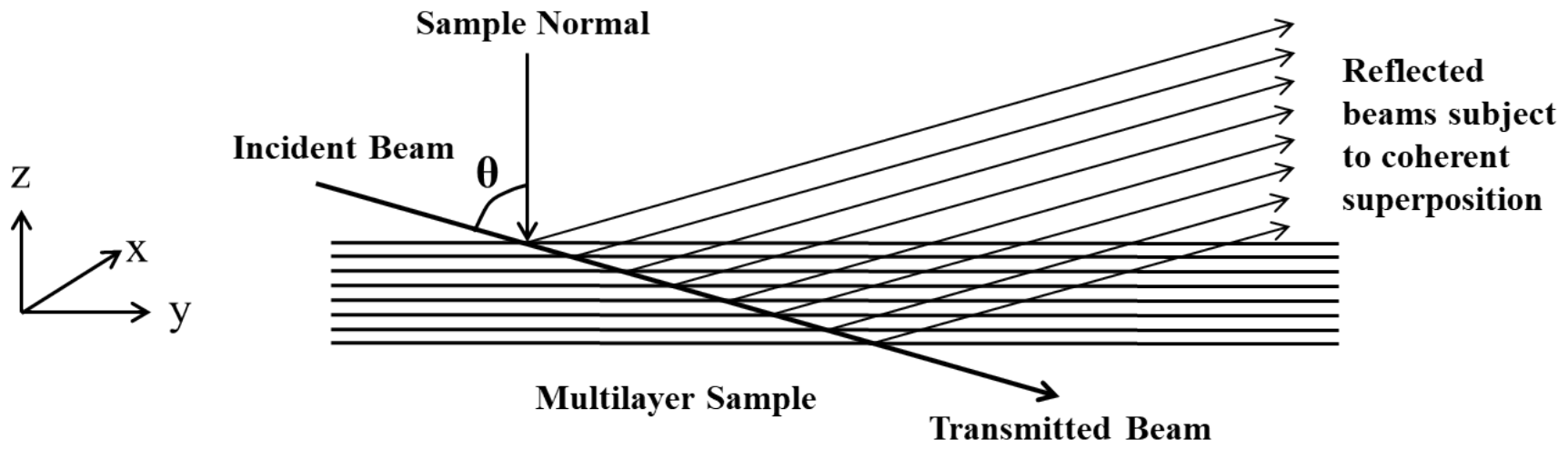

FIG. 1. $y z$ plane cross section of the scattering geometry of the scattering process. In the formalism of Zak et al. [9-12], all scattering events occur on a line parallel to the $\mathrm{z}$ axis, however in the formalism to be developed in our work, the position of the scattering events is set such that all reflected rays exit the sample at the same point. The value of the sample magnetization $\mathbf{M}$ is allowed to freely vary throughout the $3 \mathrm{D}$ sample, and the angle of incidence $\theta$ suffers slight changes according to changes in the refractive index within the sample.

interface, one may extend the formalism to a multilayer system of $N$ such layers grown on a substrate by creating a stack of such interfaces, and making use of the medium propagation matrix to move from one interface to another [9-12, 30, 32-34].

$$
\mathbf{A}_{\mathrm{i}} \mathbf{P}_{\mathrm{i}}=\mathbf{A}_{1} \mathrm{D}_{1} \mathbf{A}_{1}^{-1} \mathbf{A}_{2} \mathbf{D}_{2} \mathbf{A}_{2}^{-1} \ldots \mathbf{A}_{\mathrm{N}}^{-1} \mathbf{A}_{\mathrm{s}} \mathbf{P}_{\mathrm{s}}
$$

This expression makes use of the medium boundary matrix to define the electromagnetic field at one interface in terms of the field at a neighbouring interface after having propagated through any one of the layers of thickness $d$. The medium boundary matrix is expressed here in terms of a linear polarization basis using the notation of Qiu et al. [34], however in the work of Elzo et al. [30], where a circular polarization basis is used in which this matrix appears diagonal. It is for this reason that the circular basis is used almost exclusively when working in transmission geometry due to the absence of crosstalk between polarization states. The medium propagation matrix for the $i^{\text {th }}$ layer $D_{i}$ is defined as

$$
D_{i}=\left(\begin{array}{cccc}
U \cos \delta_{i} & U \sin \delta_{i} & 0 & 0 \\
-U \sin \delta_{i} & U \cos \delta_{i} & 0 & 0 \\
0 & 0 & U^{-1} \cos \delta_{r} & -U^{-1} \sin \delta_{r} \\
0 & 0 & U^{-1} \sin \delta_{r} & -U^{-1} \cos \delta_{r}
\end{array}\right)
$$

where

$$
U=\exp (-i k d \cos \theta)
$$

with $\theta$ representing the angle of incidence within each layer, and

$$
\delta_{i}=\frac{k d}{2}\left(Q_{n}^{y} \tan \theta+Q_{z}\right), \delta_{r}=\frac{k d}{2}\left(Q_{n}^{y} \tan \theta-Q_{n}^{z}\right) .
$$

Studying the form of the medium boundary matrix in conjunction with the scattering geometry, one notes that the propagation distance within the material appears as $d \cos \theta$, which tends towards zero at grazing incidence. This is because the formalism of $[9-12,30,32-34]$ considers the reflection events occurring in a column parallel to the $\mathrm{z}$ direction, and does not consider the absorption within the material prior to and immediately following a reflection event - absorption which occurs over a length of $z_{i} / \cos \theta$, where $z_{i}$ is the depth of the $i^{\text {th }}$ layer. In the case of incoherent multiple scattering from thick samples, a formalism was developed and experimentally tested by Dorazio et al. [43, 44], where the reflections from each interface are summed after their amplitudes and Faraday rotations are adjusted to account for the transmission within the sample before and after reflection. In the soft X-ray regime which is the main focus for this work, it appears as if a similar approach was used by $\mathrm{Li}$ et al. [19] for analysing the depth dependent domain wall profile in the skyrmion hosting multilayer system $[\mathrm{Ta}|\mathrm{CoFeB}| \mathrm{MgO}]_{N}$, however this work lacks a formal analysis section and mentions only the exponential attenuation of the reflection from multilayers deeper within the sample. Their work does however mention the increased penetration achieved from working at an incidence angle further from grazing incidence, thus allowing depth dependent information to be extracted by varying the incidence angle. Scanning the photon energy over the absorption edge can also be used for a similar effect, as will be demonstrated below in the experimental section. This simple attenuation correction can be considered a manner of applying the DWBA [45] to first order, and our approach will go one step further by considering spatial variations in the sample magnetization when calculating the attenuation and phase shift of the $\mathrm{X}$-rays passing through the sample. 


\section{SIMULATIONS: PROCEDURE AND CONSIDERATIONS}

\section{III.1. The recipe to calculate the diffraction pattern in reflection conditions}

In this subsection, we present our numerical recipe for the calculation of diffraction patterns in reflection geometry.

1. Download the atomic scattering factor files for all elements in your sample. This data may be found for all elements at the CXRO website [46], however readers must be aware that this database does not include the effects of resonance occurring when the $\mathrm{X}$ ray energy matches a core level absorption. Near to such absorption edges, one must use ones own or previously published X-ray absorption data to complement the values downloaded from the CXRO website. Simulations in this paper were performed with atomic scattering factor values measured from X-ray magnetic circular dichroism (XMCD) measurments on cobalt films at the SEXTANTS beamline at SOLEIL. Readers should beware of differing energy calibrations between different facilities, and use the measured position of the absorption edge, and not a fixed energy as the reference point.

2. From experimental X-ray absorption data, one can directly deduce the imaginary parts of the refractive indices, but to find the corresponding real parts, the Kramers-Kronig relations must be used. Their application requires the splicing of experimental XMCD data with the off-resonance refractive index/atomic scattering factor data. We performed this step assuming that at the edge of the resonant region, the refractive indices retrieved from the two sources must be equal.

3. To initialize the simulation procedure, one must have on hand a model magnetization distribution. This should be defined over the planes of each interface in the multilayer sample due to the physical significance of each interface. It is ideal in this step to use micromagnetic simulations in order to provide a physical basis to the model chosen, with a method for extrapolating a localized micromagnetic simulation over the up to several hundred micrometre size of a typical X-ray scattering experiment published previously in $[6,25,47]$. It is alternatively possible to define the magnetization distribution in geometric terms of average domain width, domain wall width and domain wall angle varying between 0 for Bloch and $\pi / 2$ for Néel. In order to proceed, one requires at this step a $3 \mathrm{D}$ vector field of the magnetization vector $\mathbf{M}$ evaluated at each $3 \mathrm{D}$ pixel in the sample under study, with the example used for simulating the subsequent experimental work shown in Fig. 2.

4. From the 3D magnetization vector field, applying equations 11 to 16 at each and every point on the $\mathrm{X}$ $\mathrm{Y}$ plane of each interface, one obtains a stack of two dimensional maps of the reflection coefficients, as is illustrated for a surface layer with Néel type domain walls in Fig. 3. Users must be careful in this case to recalculate using the appropriate angle of incidence $\theta_{e l}$ within each elemental layer el using Snell's law for an angle of incidence in free space $\theta_{i}$. To deal with the complex refractive indices, a generalized form of Snell's law was used in this work, found in Eq. 15 of Ref. [48],

$$
\sin \left(\theta_{e l}^{\prime}\right)=\frac{\sqrt{2} \sin \theta_{i}}{\sqrt{n^{2}-k^{2}+\sin ^{2}\left(\theta_{i}\right)+\sqrt{\left(n^{2}-k^{2}-\sin ^{2} \theta_{i}\right)^{2}+4 n^{2} k^{2}}}}
$$

where $\theta_{i}$ is the free-space angle of incidence, $\theta_{e l}^{\prime}$ is the angle within the layer of index of refraction $n$, and $k$ is the wavenumber.

An example of these reflection coefficients is shown in Fig. 3 for the first layer where the domain wall type is Néel, but where a longitudinal component of the magnetization is present at the points where the stripes bend.

5. Prior to and after suffering a reflection event, the X-rays which reflect from buried interfaces undergo attenuation and Faraday rotation due to transmission through a magnetized medium. The effect of this is incorporated by applying the top left quadrant of the matrix in Eq.18 to each elemental layer $e l$, however adjusting $U$ so as to include the entire optical path through each layer, and flipping sign of the direction cosine with respect to the polar magnetization for the incoming and outgoing waves.

$$
\begin{gathered}
D_{e l}^{i}=\left(\begin{array}{cc}
U \cos \delta_{i} & U \sin \delta_{i} \\
-U \sin \delta_{i} & U \cos \delta_{i}
\end{array}\right) \\
D_{e l}^{r}=\left(\begin{array}{cc}
U \cos \delta_{r} & U \sin \delta_{r} \\
-U \sin \delta_{r} & U \cos \delta_{r}
\end{array}\right)
\end{gathered}
$$

where

$$
U=\exp \left(\frac{-i k\left(d+\delta d_{r}(x, y, z)\right)}{\cos \theta_{e l}}\right)
$$

and

$$
\delta_{i}=\frac{k\left(d+\delta d_{r}(x, y, z)\right)}{2}\left(Q_{n}^{y} \tan \theta_{e l}+Q_{n}^{z}\right)
$$



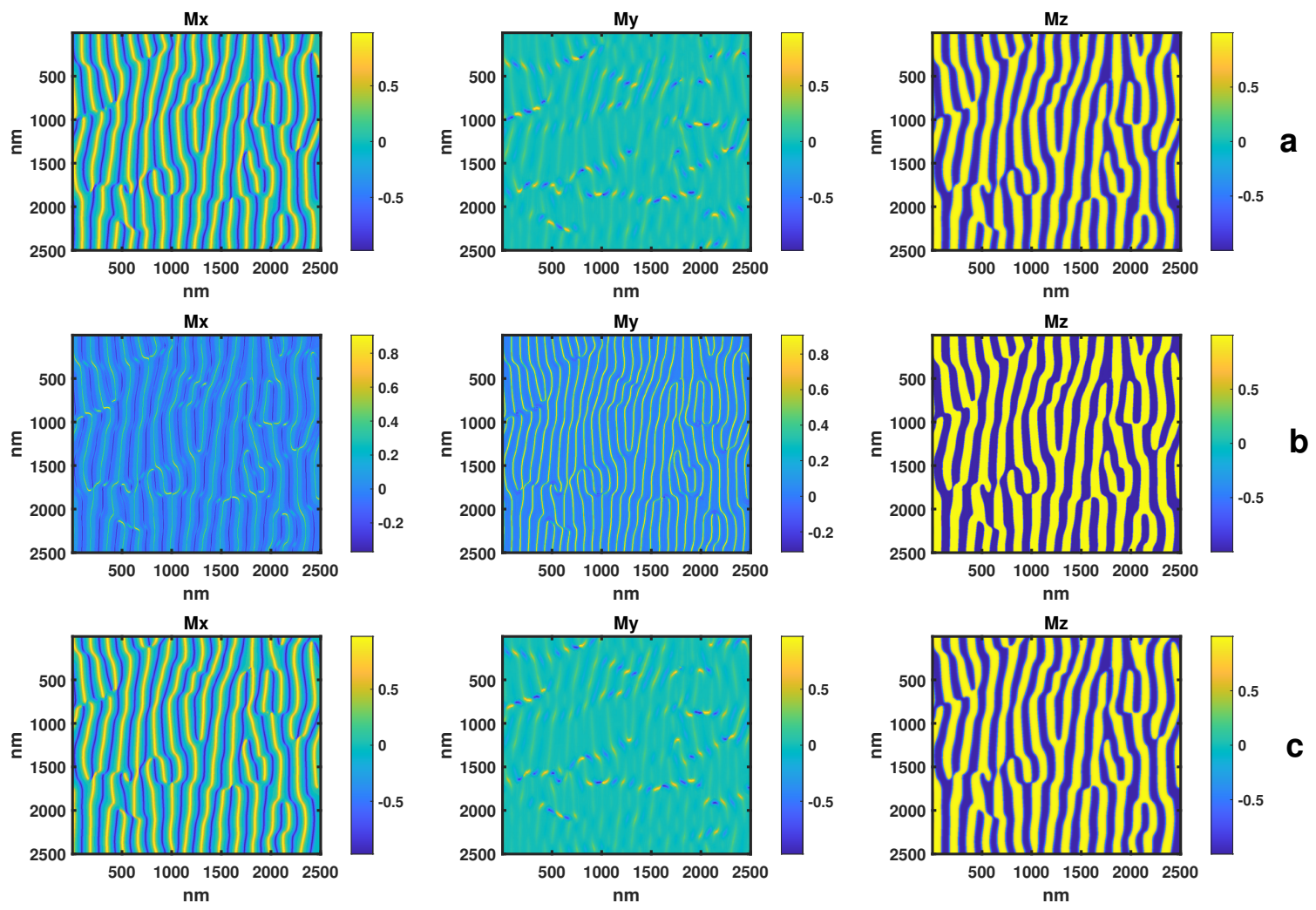

FIG. 2. a) Simulated distribution of the magnetization on the top layer of the sample, b) through the middle of the sample, and c) on the sample/substrate interface. Note the reversal of sign in $M_{x}$ between the top and bottom layers of the sample, and the fact that $M_{y}$ is dominant in the middle of the sample. This demonstrates the hybrid nature of the domain wall structure with Néel type $\left(M_{z}, M_{x}, M_{z}\right)$ on the surfaces, and Bloch type $\left(M_{z}, M_{y}, M_{z}\right)$ in the middle.
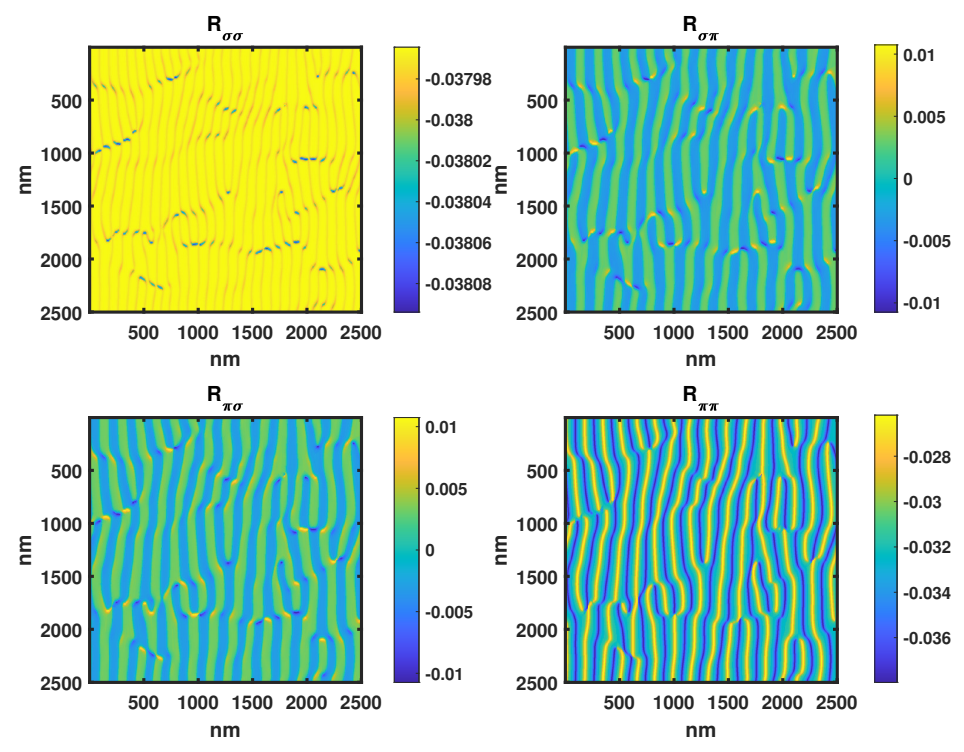

FIG. 3. Real parts of the reflection coefficients for the magnetic interface closest to the surface. The incidence angle was equivalent to the 1st Bragg angle for this thin film, and 16.5 degrees from grazing incidence. 


$$
\delta_{r}=\frac{k\left(d+\delta d_{r}(x, y, z)\right)}{2}\left(Q_{n}^{y} \tan \theta_{e l}-Q_{n}^{z}\right)
$$

where $\delta d_{r}(x, y, z)$ is the pixel by pixel deviation from a perfectly flat atomic interface caused by surface roughness - a theme which will be further discussed in the next section. For computational simplicity, it is assumed that the magnetization along the optical paths of the incoming and outgoing waves is identical. The outgoing wave reflected from interface between layer $N$ and layer $N+1$ buried within the sample, where the incident wave is given by

$$
\mathbf{P}_{\text {in }}=\left(\begin{array}{c}
E_{\sigma}^{i} \\
E_{\pi}^{i}
\end{array}\right),
$$

will therefore arrive at the surface after having been subject to the following series of matrix operators for each layer in the multilayer sample. For the non-magnetic layers, due to the fact that the $D$ matrices are diagonal, the matrix multiplication may be replaced with scalar multiplication.

$$
\mathbf{P}_{\text {out }}=\prod D_{1}^{r} D_{2}^{r} \ldots . D_{n}^{r} R D_{n}^{i} \ldots D_{2}^{i} D_{1}^{i} \mathbf{P}_{\text {in }} .
$$

In order to accurately reproduce the multilayer interference phenomena, it is necessary to calculate the phase of the incident radiation at the point of insertion relative to the phase on exit from the multilayer by calculating the optical path difference $\Delta R$. For a multilayer of different refractive indices in each layer, this may be performed in a recursive manner for each successive layer of thickness $d_{e l}$

$$
\Delta R_{n+1}=2\left(d_{e l}+\delta d_{r}(x, y, z)\right) \tan \theta_{e l} \sin \theta_{i}+\Delta R_{n},
$$

The phase shift on entry relative to the phase at exit may then be calculated as

$$
\Delta \phi=e^{\frac{2 \pi \Delta R}{\lambda}} .
$$

Readers are reminded that the phase shift of the beam as it passes through the magnetized sample is also accounted for by Eq. 28.

6. After summing the phase shifted and attenuated wavefields from each interface within the sample at the level of the surface, the resulting total wavefield may be propagated to the detector using standard free space propagation. The effective combined reflection coefficients representing the phase matched sum of all reflections are shown in the Fig. 4, and may be used to calculate the outgoing wavefield given an incoming wavefield. One should be aware that the application of a simple Fourier transform for the freespace propagation gives an acceptable performance in situations where magnetic domains are aligned parallel to the scattering plane, however does not reproduce exactly the experimentally observed diffraction from features perpendicular to the scattering plane. This is due to Ewald spehere curvature, and causes the effective angle of incidence to depend upon the value of $q_{y}$ in the resulting diffraction pattern. Variations of the effective angle of incidence with $q_{y}$ increase as the angle of incidence approaches grazing incidence.

For a 20 layer multilayer sample and a 1024x1024 array, the computation time with MATLAB on a Hewlett Packard Workstation with 12 cores from the year 2014 was approximately 7 minutes. In the case where it is sufficient to simulate with perfectly aligned stripe domains of identical size, speed can be increased by using a much smaller array size.

\section{III.2. Introduction to the theme of Roughness in Simulations}

The above described algorithm when $\delta d(x, y, z)=0$ deals with the ideal situation where the sample can be described as a regular stack of perfectly smooth atomic layers. In practice however, such stacks do not exist, and one must take into account the influence of roughness and irregular spacing of the atomic layers. A dimpled surface in the $\mathrm{X}-\mathrm{Y}$ plane will produce a diffuse scattering background or coherent speckle depending upon the coherence of the incident radiation. This diffuse scattering will appear underneath and possibly interfering with any magnetic peaks detected in the scattering pattern; and, especially at low spatial frequencies will also weaken the interference effects between the different layers in the sample. A full treatment of surface roughness can be found in the Refs. [30, 42, 49, 50], where the treatment was statistically based upon a Gaussian distribution of the spatial deviations from a perfectly flat multilayer stack. In this work, because our algorithm explicitly generates a multilayer stack with a given magnetic domain pattern, we account for this roughness directly by adjusting the $z$ spacing between each layer on a pixel by pixel basis according to a random roughness function $\delta d_{r}(x, y, z)$ of amplitude and planar spatial frequency distribution which best reproduce the observed experimental background.

During the transmission through the sample before and after a reflection event, it is also likely that there are further losses not accounted for by the imaginary part of the refractive index due to diffuse scattering from grain boundaries. Such diffuse scattering was observed in previous work [6,25], and is of increasing importance as the incidence angle approaches 90 degrees (as measured from the normal). The magnitude of this effect is expected to depend on photon energy, angle of incidence, and specific sample fabrication characteristics; and we are of the opinion that a full investigation of this factor is warranted. We note that the custom thus far of subtracting the experimental diffuse background by cubic spline fitting or similar also includes the implicit assumption that the diffuse background and magnetic 

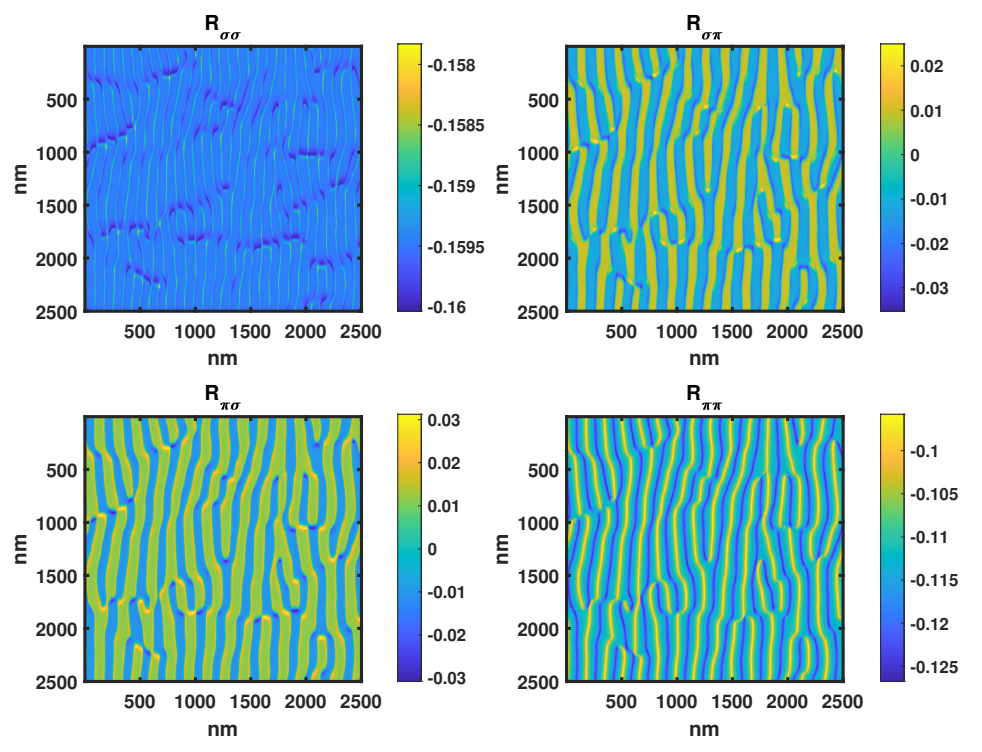

FIG. 4. Real parts of the total effective reflection coefficients entire thin film. The array of effective reflection coefficients shown here is the sum of phase matched reflection coefficients for each of the magnetic interfaces in the sample. The incidence angle was equivalent to the 1st Bragg angle for this thin film, and 16.5 degrees from grazing incidence.

scattering signal add incoherently. Such an assumption is however not valid, as is demonstrated by the fact that Fourier transform holography [51, 52] of magnetic samples in transmission geometry is an established field, and depends upon the interference between the magnetic signal and the non-magnetic reference signal. The effects are however small in cases where the magnetic scattering signal is much stronger than the diffuse background, and where the spatial coherence length is much less than the spot size; conditions which are met for most XRMS experiments.

\section{III.3. Transmission Geometry}

In most experimental cases of interest where scattering is to be used, reflection geometry is a more sensitive probe due to the strong differential contrast between the different polarization states. Transmission geometry scattering, which was studied elsewhere in more detail $[6,25]$ is however of interest particularly where one is working with single layer samples where reflection would only occur from the top surface (and in some cases from the substrate), or where an imaging type experiment is proposed such as that of Donnelly et al. [53]. In transmission geometry it is necessary to rotate the sample in order to extract 3D information about the sample, whereas in the case of reflection geometry, one may tune the X-ray energy on and off resonance to enable differing amounts of depth sensitivity. For this configuration, the simulated sample should be defined on a cubic matrix, which must then be interpolated in a series of diagonal slices perpendicular to the vector of propagation of the incident beam and subject to multiple slice propagation[54]. Working in a circular polarization basis, the refractive index is given by utilizing Eq. 7, allowing the complex transmission function for the projection over a given slice of thickness $\delta x$ to be expressed as

$$
T=\exp \left(\frac{-2 \pi}{\lambda} i\left(n_{0}\left(1 \pm \sigma g Q_{n} / 2\right)-1\right) \delta x\right)
$$

This process accurately reproduces the deviations from simple projection approximation modelling observed in experiments due to curvature of the Ewald sphere, and enhanced absorption of diffracted X-rays in the direction towards the sample due to an increased optical path length within the sample. Once clear of the sample, Fourier transform based free-space propagation may be used to propagate to the detector plane, with no caveats as in the case of reflection geometry, due to the wavefield being defined over a plane parallel to the direction of beam propagation. It was our original intention to use this multiple slice propagation as the approach for the reflection geometry work presented here, adding reflection events at each interface as the beam passed through the sample. This was however found to be both numerically unstable due to observed "diffraction" from the pixel matrix, and extremely slow due to the large number of Fourier transforms which needed to be evaluated. As a result, this multi-slice propagation approach was discarded for our approach based on projection approximation transmission and a single reflection event per reflected beam. 


\section{III.4. Treatment of X-ray coherence}

Levels of spatial coherence of synchrotron sources have been steadily increasing, with the newest diffraction limited sources expected to provide close to full coherence over the size of the beam. In the case of full coherence, the task of simulating the diffraction is straightforward: The Huygens Fresnel principle permits one to calculate diffraction via Free-Space propagation of the outgoing wavefield (in both reflection and transmission geometry). In the case of partially coherent illumination, the most straightforward manner in which to simulate the resulting diffraction is via the coherent mode expansion [55], which in the case of a disordered and statistically stationary sample may be approximated by summing the results of a set of realizations of the code assuming a coherent beam over the region of each simulation each simulation performed with a different real-space domain configuration. This latter approach was used in an approximate manner by Flewett et al. in Ref. [25], simulating partial coherence by summing a series of 10 realizations of the simulation with coherent radiation, but using a different matrix of random numbers with which to simulate the magnetic domain pattern. This same approach will be used here for the simulations where a disordered magnetic domain pattern is used, and in the case where roughness is present.

\section{EXPERIMENTAL VALIDATION}

The approach described in the previous section was developed to respond to need of analysing the X-ray reflective scattering data of Pianciola et al. [23] (in the case of a single layer), and subsequently those submitted for publication in parallel with this manuscript [22, 24], where the approach was generalized to a multilayer system, and the DWBA approach introduced. The work of Burgos Parra et al. [22] deals with a stripe domain system with an external field applied along the length of the stripes, and the work of Léveillé et al. [24] an antiferromagnetic worm domain system where the magnetic signal is strongest at $1 / 2$ and $3 / 2$ of the multilayer Bragg angle. In contrast with the above cited examples where the general aim of their work was to use XRMS to study the magnetic properties of the sample, the experimental tests presented here were designed with the explicit aim of probing the robustness of our theoretical framework.

\section{IV.1. Experimental and Simulation Conditions}

Our tests were performed using the same $\mathrm{Ta}_{1} \mid \mathrm{Pt}_{0.8}$ $\left[\mathrm{Co}_{0.8}\left|\left(\mathrm{Al}_{2} \mathrm{O}_{3}\right)_{1}\right| \mathrm{Pt}_{1}\right]_{20 r e p} \mid \mathrm{Pt}_{2}$ multilayer as in the work of Burgos-Parra et al (numbers in subscript being thicknesses in nm except the 20 which is the number of repetitions). [22]. The XRMS measurements were performed at the RESOXS diffractometer [56] at the
SEXTANTS beamline[57] of the synchrotron SOLEIL in France. They were conducted in reflection geometry with both circular polarizations at energies between 775 and $781 \mathrm{eV}$, with the diffracted X-rays collected using a Peltier-cooled square CCD detector covering $6.1^{\circ}$ at the working distance of this study. For all measurements the exit slits were set at $25 \mu \mathrm{m}$ with a resolving power around 5000, and total exposure times were $5 \mathrm{~s}$ at the first Bragg angle, 15s for the second Bragg angle and 100s for the third Bragg angle. For seeding our simulation XRMS code, micromagnetic simulations were performed using the energy minimization procedure implemented in Mumax3 to generate a domain wall profile [14]. The parameters used were $A=9.5 \mathrm{pJm}^{-1}$ for the Heisenberg exchange, $D=1 \mathrm{mJm}^{-2}$ for the Dzyaloshinskii-Moriya Interaction (DMI) constant, $K u=1.43 \mathrm{MJm}^{-3}$ for the uniaxial anisotropy along the $\mathrm{z}$ axis and $M s=1.37$ $\mathrm{MAm}^{-1}$ for the saturation magnetization of the cobalt layers. A cross section of a single domain wall obtained with the simulation is shown in Fig. 5a, where the skew structure in the out of plane direction typical of a nonzero DMI [17] can be appreciated. In Fig. 5b, we show the specular reflection at $778 \mathrm{eV}$ as a function of incidence angle, from which we determined the three Bragg angles at which subsequent measurements were made. It should be noted that for experimental measurements, the Bragg angles found via specular reflection were used, whereas for simulations we used the Bragg angles calculated from the nominal sample thickness. The small difference between the two (of $1^{\circ}$ for the 1st Bragg angle) is due to a slight calibration imperfection in the sputtering system. In Figs. $5 \mathrm{c}$ and $\mathrm{d}$ we show values of $\delta$ and $\beta$, the deviations from unity of the refractive index $n$ as a function of incidence energy. In $5 \mathrm{c}$ we show the values of non magnetic part caused by fluctuations in $f_{c}^{n}$ and in $5 \mathrm{~d}$, the values corresponding to the magnetic part caused by fluctuations in $f_{m 1}^{n}$. In Fig. 5e, we show panels of the raw scattering pattern at $778 \mathrm{eV}$ for each Bragg angle (without correcting for the projection angle in the $q_{y}$ direction). For subsequent analysis, this data was integrated over the $q_{y}$ direction, and the integrated intensities were calculated as with powder diffraction by first subtracting a cubic spline estimate of the diffuse background curve. This integration over the $q_{y}$ direction was made possible due to the fact that we are working with a stripe domain sample. Had a worm domain or highly disordered stripe pattern been used as in [24], such integration results in a loss of information.

Our tests were the following: 1. The examination of the variation of the scattering pattern moving off the multilayer Bragg angle in order to evaluate the importance of interference effects beyond the well known reduction of signal strength due to the loss of constructive interference. This not only served the role of uncovering new physics, but also for verifying the correctness of step 5 of our algorithm - the part related to the phase matching between the beams reflected from different layers. 2. Energy scans over a $6 \mathrm{eV}$ range across the resonance at the first three multilayer 
Bragg angles to evaluate the suitability of our algorithm for extracting depth sensitive information about the magnetization distribution, and to evaluate the influence of interface roughness and poly-crystalline structure on the experimental outcome.

\section{IV.2. Interference effects moving off the multilayer Bragg Angle}

It is known that working at the multilayer Bragg angle is advisable in order to increase the signal to noise ratio, however when moving off the Bragg angle not only is the diffracted intensity reduced, but major qualitative changes in the scattering signal are observed. In the first test with results in Fig. 6, we looked both on the first multilayer Bragg angle, and $1^{\circ}$ off the Bragg angle towards grazing incidence, both at zero field, and with an external field of 1900 Gauss in-plane along the $\vec{y}$ direction applied.

In Fig. 6, in addition to the expected signal reduction observed moving off the Bragg angle, there are three features which readers should take note of: 1 . The degree of asymmetry present in the first order peaks both with and without external field defined as $\left(I_{l}-I_{r}\right) /\left(I_{l}+I_{r}\right)$ increases by approximately 20 percentage points as one moves off the Bragg angle. 2. The dichroism observed in the second order peaks where field is applied is much stronger on the Bragg angle compared with being off the Bragg angle, and 3. The experimental diffuse background in the case with field applied is approximately double in one polarity compared with the other. This dichroism has been observed in specular reflection [35, 36], and one may consider the background to be due to roughness and scattering in transmission from the poly-crystalline grain walls causing broadening of the specular peak.

The theoretical results were simulated assuming a RMS interfacial roughness of 0.7 Angstroms with a Lorentzian profile, and with the imaginary part of the refractive index $\beta$ increased by $\Delta \beta=0.001$ to approximate losses due to diffuse scattering in transmission. Each theoretical plot was generated as the ensemble average of 10 simulations for different randomly generated magnetic domain patterns similar to those shown in Fig. 2. Here in Fig. 6, the observations regarding the changes in the first and second order peaks can be explained by interference between the different waves emitted from each interface in the multilayer sample, with the increase in observed asymmetry of the first order peak arising from the change of sign in the asymmetry of the reflected waves from the inferior layers in the sample where the chirality is opposite of the superior layers [17, 19]. Conceptually, when the sample is aligned at the Bragg angle, at the detector we have constructive superposition between the left-right asymmetry diffraction patterns from the top layers of the sample, the zero asymmetry from the middle layers with a Bloch domain wall, and right-left asymmetry from the contributions from the inferior layers. The sum of these gives us a measured scattering pattern with moderate asymmetry, the final value of which depends upon the relative amplitudes of the contributions from each layer. When we move off the Bragg angle however, there is a phase shift between the contributions from each layer, and when the contributions from the lower layers of the sample with the opposite asymmetry to the top layers are superimposed out of phase, the result is not a reduction in the asymmetry but rather a reduction in the intensity and an increase in the asymmetry. At zero applied field, we note that the 3rd order diffraction peak is much weaker in the experimental compared with the theoretical plot. This could be due to a greater degree of stripe disorder in experiment compared with theory, or due to the presence of narrower Bloch walls causing a sharper transition between up and down magnetization in the middle layers of the sample. A reduction of the width of the Néel caps can however be ruled out, because that would reduce the degree of asymmetry - something not observed in this case.

In order to simplify the analysis in subsequent sections, in future plots we will express the measured diffraction pattern as a set of integrated intensities representing the area under each diffraction peak - as with powder diffraction. Neglecting however the interference between ordered magnetic scattering and the diffuse background could introduce errors which increase as the strength of the background increases relative to the magnetic signal.

\section{IV.3. Born Approximation vs. Distorted Wave Born Approximation: Explanation of Dichroism observed with Applied Longitudinal Field in Second Order Scattering Peaks}

In the previous section, a notable dichroism was observed in the second order scattering peaks when observed at the Bragg angle - a phenomena which is extensively studied by Burgos Parra et al. in their work developed in parallel with this manuscript [22]. In theoretical terms, this dichroism at second (and fourth) order was not explained solely by the spatial differences in the reflection coefficients across each plane in the multilayer, but rather only by including the differential absorption in transmission according to the incident polarization and magnetization vector. In fact, it was the need to explain this second order dichroism in the work of Burgos Parra et al. [22] which impulsed the addition of the DWBA to the simulation recipe discussed here - an addition which approximately doubled the computation time. This is demonstrated in Fig. 7 theoretical curves are shown for both the Born Approximation case and the DWBA case (with the intensity scale shortened to highlight the difference in the second order peaks, with no important difference observed at 1st order). Only in the DWBA case was it found possible to reproduce the dichroism observed experimentally between the two polarization states at second order. The nature of the dichroism at second and fourth order is distinct from that at first and third order: At odd orders, we observe 

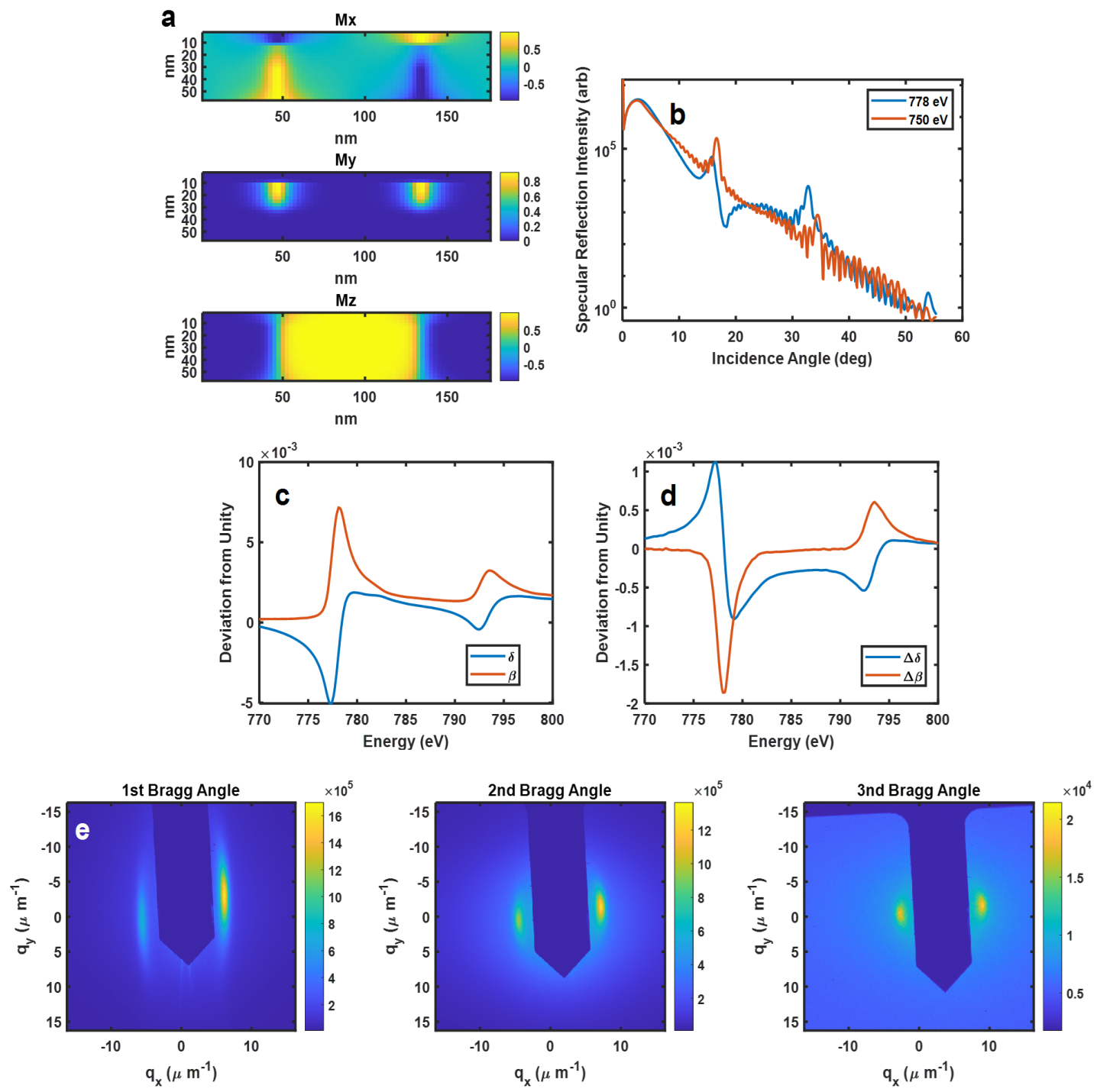

FIG. 5. (a)Cross sections in the $\mathrm{x}, \mathrm{z}$ plane through one ideal magnetic unit cell used for generating the simulations. Note the Bloch wall being situated in the upper part of the film. (b) Specular reflection curves both on resonance (778 eV) and off resonance $(750 \mathrm{eV})$. (c) $\delta$ and $\beta$ values for cobalt used in the simulations (measured at SEXTANTS beamline of Soleil). (d) The magnetic deviations of the refractive index dependent upon the angle between the magnetization and the Poynting vector of the incident light. (e) Raw XRMS measurements for the 1st, 2nd and 3rd Bragg angles at the incidence angles determined by the specular reflection scan $\left(15.5^{\circ}, 33^{\circ}\right.$ and $\left.53.8^{\circ}\right)$.

the characteristic left/right asymmetry which flips with a change of polarization, however at even orders we observe left/right symmetric diffraction peaks whose intensity depends upon the polarization. From a semantic point of view, it is perhaps not appropriate to be talking about a pure reflection configuration in this case, but rather a situation where reflection and transmission are combined - the strong second order peaks observed due to the Bloch walls being evidence of the transmission component at play. It will be interesting in the near future to combine reflection with transmission measurements in order to obtain a more precise quantification of the size of the Bloch walls - and also obtain a better quantification of the role of the poly-crystalline nature of the sample in the generation of the background signal. The explanation of this dichroism in the second order peaks, and the conclusion here that it is due to differential transmission along the Bloch walls and not merely due to differences in the reflection coefficients, is one of the chief conclusions of this work.

\section{IV.4. Studying Depth Penetration with Energy Scans at Different Bragg Angles}

This work, inspired by that of $[19,26]$, looks to evaluate the extraction of information in the $\vec{z}$ direction, hopefully extending from the qualitative treatment of $[19,26]$ to a more quantitative treatment. A further inspiration was based on the work of Legrand et al.. [17], where the 

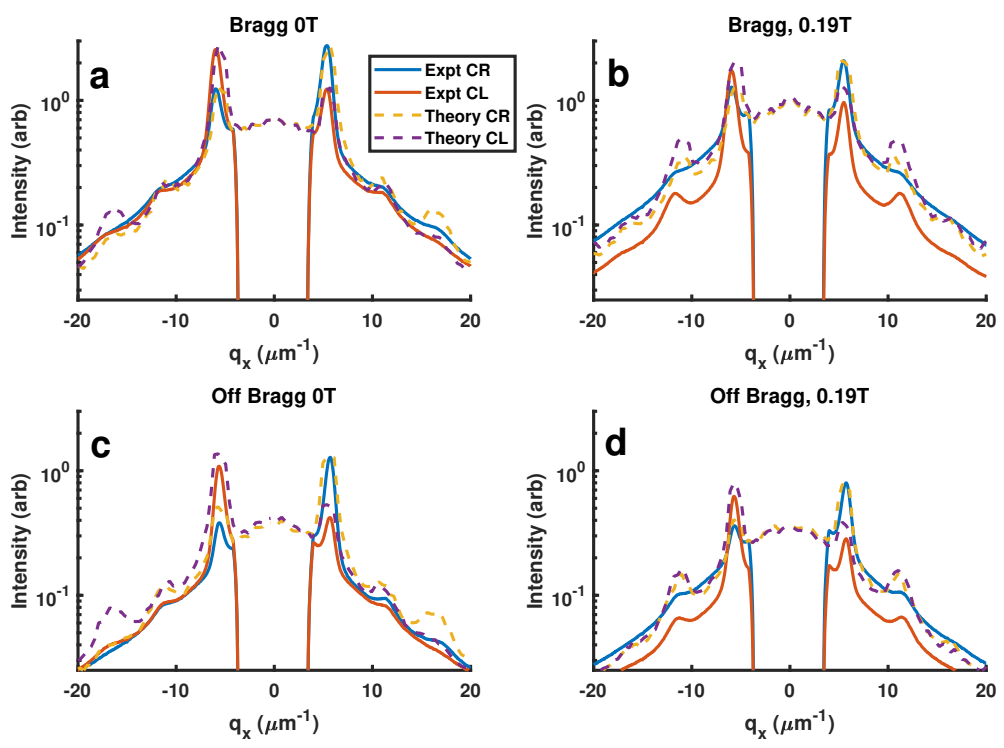

FIG. 6. Experimental scattering data (integrated over the $q_{y}$ direction) with and without an in-plane applied field on and off the multilayer Bragg angle. The four lines on each graph represent left and right polarized light for both experiment (solid) and theory (dashed) lines. In (a) we show the results at zero field on the Bragg angle, in (b) at a field of 0.19T on the Bragg angle, in (c) at zero field but $1^{\circ}$ off the Bragg angle, and in (d) at $0.19 \mathrm{~T}$ and $1^{\circ}$ off the Bragg angle. Note the logarithmic intensity scale
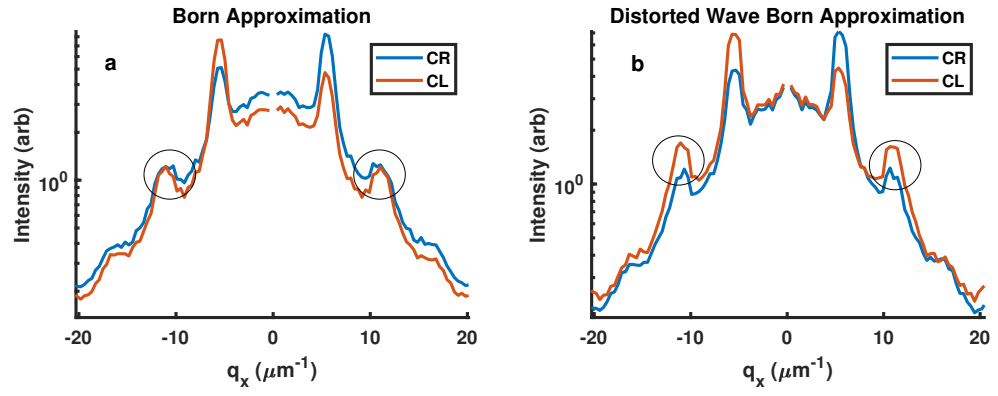

FIG. 7. Simulated scattering scans with a 0.19T external field applied along the magnetic stripe axis (a) using the Born Approximation accounting for the reduced contribution from buried interfaces due to X-ray absorption, and (b) using the Distorted Wave Born Approximation as presented in the text. The plots show the simulated scattering summed over the $q_{y}$ direction, with the second order peaks highlighted to show the difference between using the BA vs. the DWBA.

z position of the Bloch wall was found to be directly related to the value of the DMI. Should one be able to quantitatively determine the value of the Bloch wall, then it could be possible to determine the DMI by means of resonant X-ray scattering, complementing the existing techniques, notable Brillioun light scattering [58, 59] or domain wall creep [60]. As will be seen in the following results and accompanying discussions, the full quantification of the real effective penetration depth will be necessary before a quantitative measurement of the DMI can be made.

For this part of the investigation, we measured the resonant scattering data at the first three multilayer Bragg angles of our hybrid Néel/Bloch/Néel domain wall sample, with the incidence angles determined by the specular reflection scan shown in Fig 5, and at each angle scanned the energy from 775 to $781 \mathrm{eV}$ in $0.2 \mathrm{eV}$ steps without the application of an external field. The data was then integrated over $q_{y}$, and the integrated peak intensities were calculated after subtracting the background using a cubic spline fit. These curves can be seen in Fig. 8, along with the associated values of the asymmetry ratios. (The jaggedness of the experimental asymmetry ratio curve for the third Bragg angle is due to the poor signal to background ratio). In this figure, the asymmetry can be seen to reduce with increasing penetration within the sample, with a change of sign observed at the third Bragg angle - this change of sign due to the opposite chirality of the inferior layers of the sample. It is the position of this change of sign as a function of penetration depth which can be used to determine at which point the handedness of the Néel 

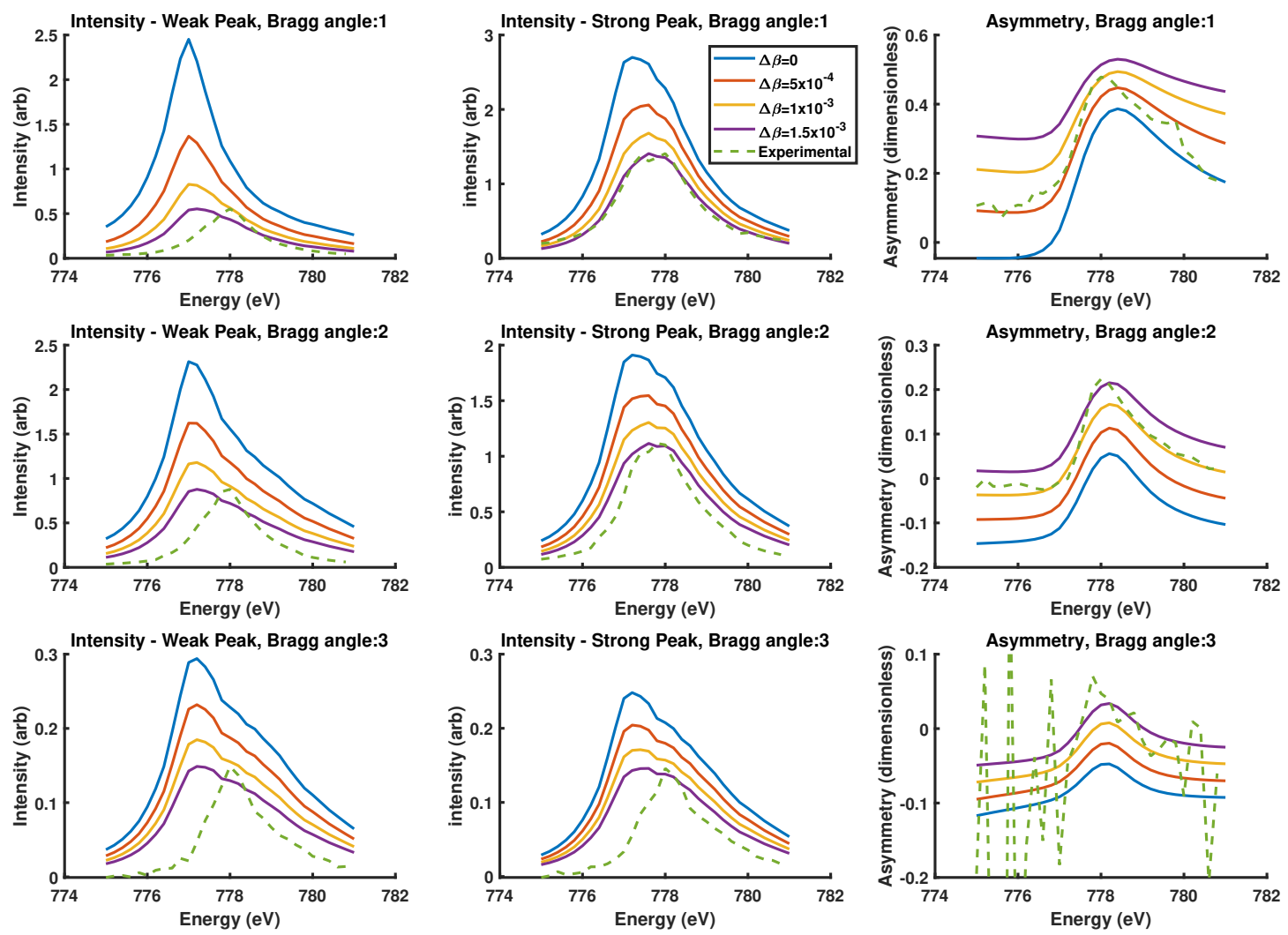

FIG. 8. Energy scans of the asymmetry ratio without roughness used in the simulations. Results for the 1st multilayer Bragg angle $\left(16.8^{\circ}\right)$ in the top row, the 2nd multilayer Bragg angle $\left(35^{\circ}\right)$ in the middle row, and in the bottom row the 3rd Bragg peak at $58.8^{\circ}$. Theoretical curves are shown in solid lines for different values of the poly-crystalline structure induced change in the imaginary part of the refractive index $\Delta \beta$, and the experimental data is shown in a dashed line. The intensities of the "weak" peaks refer to the less intense scattering peak at one helicity, and the corresponding less intense peak from the opposite side as measured with the opposite helicity. The "strong" peak intensities refer to the opposite more intense scattering peaks.

component of the domain wall flips, and thus realise an estimation of the DMI [17].

Successfully modelling the experimental curves observed in this figure would confirm the proposed magnetic domain profile shown in Fig. 5, and would therefore represent a major advance in the field. The theoretical curves on this figure do not consider interfacial roughness, and for computational speed were performed using an ordered stripe pattern (instead of the disordered pattern used in Figs. 6 and 7). The four theoretical curves shown in Fig. 8 are for increasing levels of increase of the imaginary part of the refractive index $\Delta \beta$ due to charge scattering from the grains in the sample. We know from the observation of diffuse charge scattering in transmission geometry in previous work $[6,25]$ that this value of $\Delta \beta$ is non-zero, and electron microscopy work on a similar sample by McVitie et al.[61] found an average grain size of $4 \mathrm{~nm}$. Additionally, during the transmission geometry beamtime leading to Ref. [6], a long exposure normal incidence measurement was made whilst searching for higher order magnetic diffraction peaks which revealed a charge scattering SAXS ring corresponding to an average grain size of $8 \mathrm{~nm}$. A precise measurement of losses due to diffuse scattering by the poly-crystalline grains by X-ray absorption measurements in transmission geometry as a function of incidence angle and energy remains however to be done. The strong relation between the transmission loss and the measured scattering signal is an indication that this matter will need to be resolved before a detailed characterization of the internal magnetic structure can be resolved.

As can be seen, as the degree of transmission loss due to diffuse scattering from the poly-crystalline grains increases, the peak of the intensity spectra moves from $777 \mathrm{eV}$ towards the experimental peak at $778 \mathrm{eV}$, but does not reach this point. The peak of the resonance is at 778 $\mathrm{eV}$, and for a single layer this is where the reflection signal would be expected to be strongest. However as one moves off the resonance, the transmission increases which in the presence of a perfect multilayer stack drastically increases the reflection when measured on the Bragg angle. It 

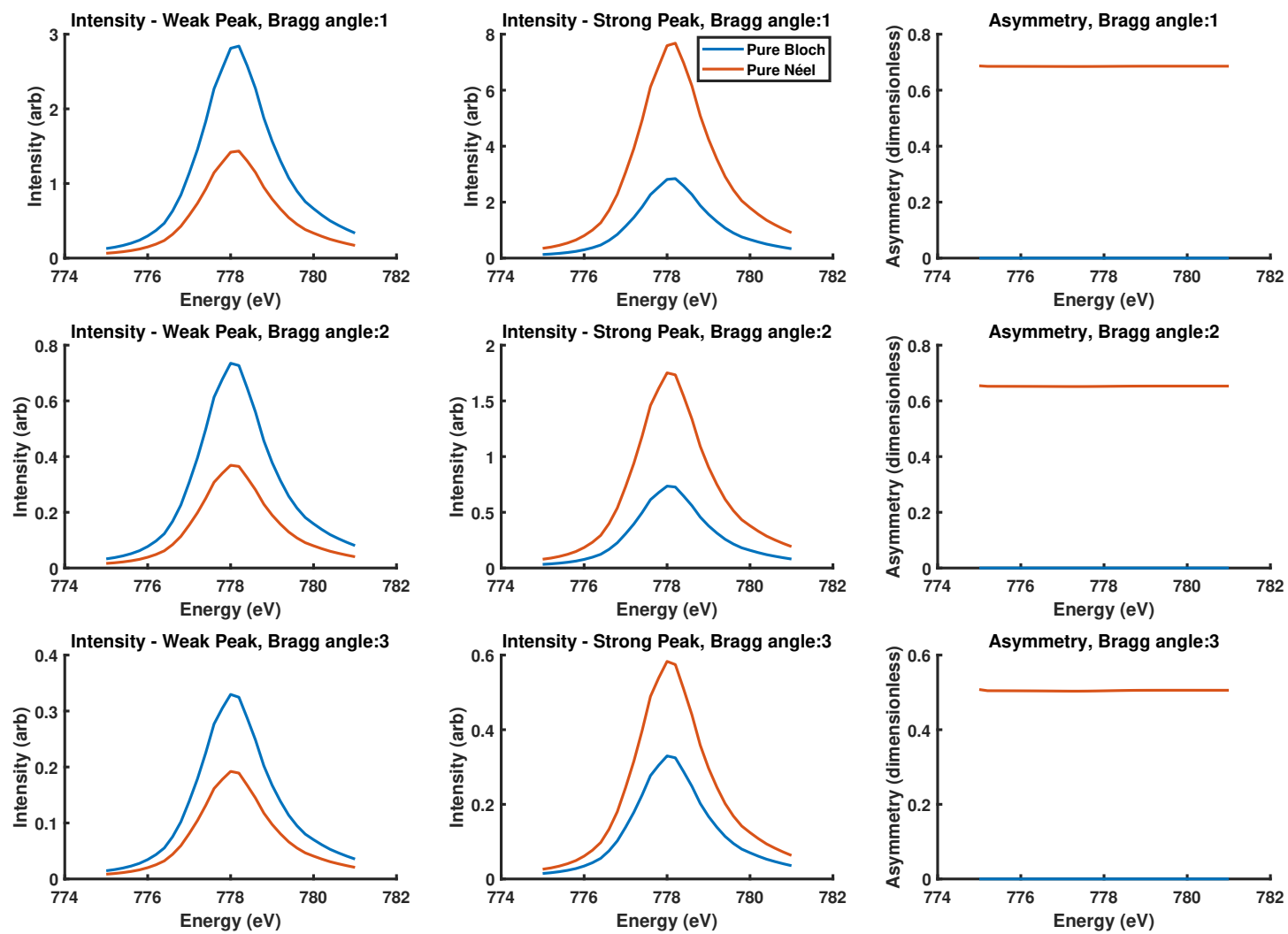

FIG. 9. Energy scans of the asymmetry ratios and scattering intensities for a hypothetical perfectly smooth interface for ordered stripe domain pattern with either pure Bloch or pure Néel domain walls. Each row represents the incidence angle equal to the 1st, 2nd or 3rd Bragg angle corresponding to the multilayer sample studied in this paper. The intensities of the "weak" peaks refer to the less intense scattering peak at one helicity, and the corresponding less intense peak from the opposite side as measured with the opposite helicity. The "strong" peak intensities refer to the opposite more intense scattering peaks

could be that the local periodicity of the multilayer stack is locally imperfect, and this could be investigated in a future experiment studying the speckle from a coherent illumination experiment on a magnetically saturated sample, both at high levels of photon flux with a beamstop to study high spatial frequency roughness, and at low flux without a beam stop for the low spatial frequency roughness which is could be responsible for the weaker than predicted Bragg interference.

For purposes of comparison, we evaluated the scattering patterns for a perfectly smooth single magnetic interface, with domain wall periodicity and domain wall width equal to those on the surface layer of the sample under study. In this case however, we assumed that the domain wall type was either pure Néel or pure Bloch. These results can be seen in Fig. 9

In Fig. 9, readers should note that the maximum scattering intensity is found at $778 \mathrm{eV}$, and not at $777 \mathrm{eV}$ as was seen for the multilayer simulations, further evidence that we are most likley overestimating the effects of constructive interference on the Bragg angles in our simulations. Also, we note that for a pure Néel domain wall that we have an asymmetry ratio greater than 0.6, whereas for a pure Bloch wall the asymmetry ratio is zero. An intereseting corollary can be seen when comparing the case of the pure Néel wall and the measurement performed off the Bragg angle for the Hybrid sample shown in Fig 6. In this case, the observed asymmetry ratios are very similar, and it is imaginable that one could mistake a hybrid domain wall sample for a pure Néel sample if only a single measurement is made on a sub-optimally aligned sample. A simple energy scan or rocking curve measurement will however clear any ambiguity in that domain. Comparing Figs. 8 and 9, we note that in the case of Fig. 9, that the asymmetry is independent of the photon energy. The reason for this is that the photon energy is a related to the absorption length, and therefore the depth over which the X-rays are probing the magnetic structure of the sample. For a sample where the magnetization is independent of the depth within the sample, it is therefore to be expected that the asymmetry be independent of the energy. This is not the case however with the magnetic scattering intensity, which depends upon the value of the magneto- 

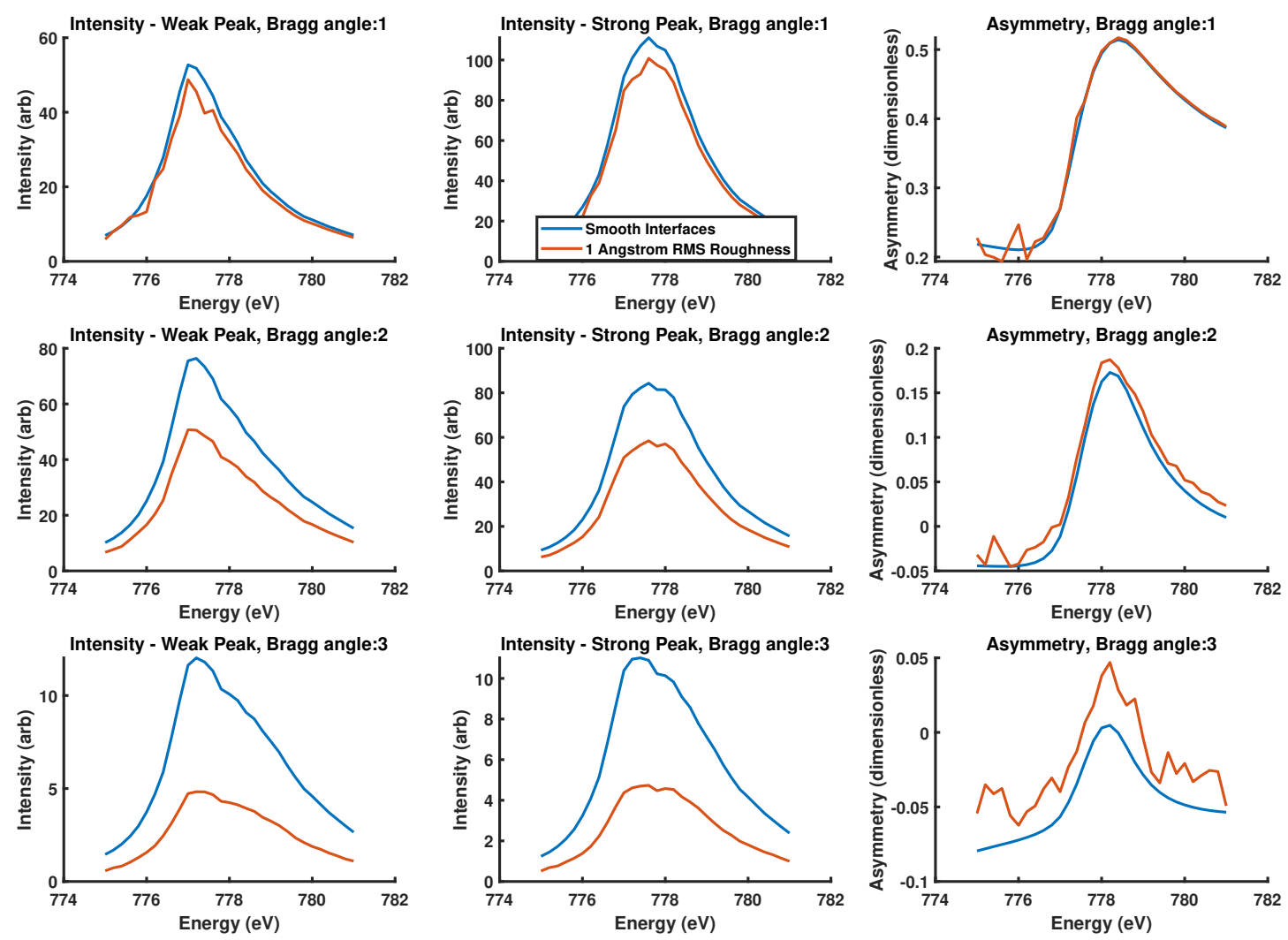

FIG. 10. Energy scans of the asymmetry ratio with 1 and 2 Angstrom RMS roughness used in the simulations. Results for the 1st multilayer Bragg angle in the top row, the 2nd multilayer Bragg angle in the middle row, and in the bottom row the 3rd Bragg peak. Each line represents a differing level of interfacial roughness. The intensities of the "weak" peaks refer to the less intense scattering peak at one helicity, and the corresponding less intense peak from the opposite side as measured with the opposite helicity. The "strong" peak intensities refer to the opposite more intense scattering peaks.

optical parameter $Q$, which in turn depends upon the energy-dependent magnetic part of the atomic scattering factor $f_{m 1}^{n}$.

We also compare the energy scan simulation without interfacial roughness (but including an increment in the absorption due to diffuse scattering from grain boundaries $\Delta \beta=0.001$ ), a with interfacial roughness of 1 Angstrom in Fig. 10. In this case, the simulated results shown are the mean of 100 different simulations with random roughness distributions, however for speed, the magnetic domain pattern remained a perfect stripe pattern as in Fig. 8. In this present case, a Lorentzian roughness spectrum was used, which is relatively close to the experimental data as seen in Fig. 6. It can be seen here that the overall behaviour of the (background subtracted) peak intensities for the simulated rough sample is similar to that of the ideal sample, except for an expected reduction in the scattered intensity. We note that a small systematic bias towards higher asymmetry ratios is observed for the case where roughness is present, however the effect is small, suggesting that except in cases with very high background that one is justified in not considering interference between charge and magnetic scattering when subtracting the background from experimental data. We note as well that the amount of signal to background ratio is best at the 1st Bragg angle and gets steadily worse as we move to the second and third Bragg angle, as evidenced by the greater amount of residual noise present even after summing 100 the results from 100 different roughness distributions. This is observation of poorer signal to background is consistent with experimental results as shown in Fig. 5e. Due to the need to sum a large quantity of diffraction patterns to obtain adequate statistics in order to generate this figure, we increased the pixel size to $8.75 \mathrm{~nm}$ (20 pixels per domain period), and calculated over a 60x60 array. This therefore excludes the study of coarser spatial frequencies in the roughness spectrum - a contribution which could be key for understanding why our simulations consistently overestimate the role of constructive Bragg interference at energies below the resonance. 


\section{CONCLUSION}

In the course of this work we have presented a general numerical algorithm for simulating magnetic scattering from an arbitrary 3D magnetized, multilayer sample, based upon a generalization of the formalism of Zak et al. $[9-12]$ in the DWBA. The algorithm was developed as a tool for the analysis of colleagues' data with the aim of creating the most general framework possible [2224 ], with the further experimental tests presented in this paper devised to evaluate the algorithm's robustness. In these experiments we succeeded in achieving agreement between theory and experiment as the angle of incidence moves on and off the Bragg angle, and we also succeeded in explaining the dichroism observed in the second order Magnetic scattering peaks due to transmission through and parallel to the Bloch walls before and after the reflection event. This explanation of the dichroism of the second order peaks is key to the work of Burgos Parra et al, where the evolution of the 3D magnetization morphology is studied as a function of applied field. Further work in the area of interfacial roughness and scattering in transmission from polycrystalline grain walls will however be necessary to fully explain the behaviour observed as the depth penetration is varied, and will be necessary before a fully quantitative determination of magnetic structures is possible.

The long term aim of this investigation is to extract a plausible range of magnetic structures given a set of experimental data - most likely via an iterative algorithm. There is still much work to arrive at this point, such as the optimization of an experimental protocol for the choice of measurements (energy and incidence angle), a revision of the role of micromagnetic simulations in the process, and the selection of the optimal computational algorithm for implementing the iterative process. The most significant advances presented in this present work are in our the opinion the generalization of the Zak[9-12] formalism for non-specular reflection, the addition of the DWBA allowing the study of Bloch domain walls, and the presentation of a step by step algorithm allowing for the scattering to be calculated from an arbitrary distribution of the magnetization. This work is not restricted to static magnetic configurations, it can obviously be applied to time-resolved experiments, and under different field or temperature conditions. Further benefits will also come from the highly coherent 100nm spots available from new diffraction limited storage rings, which will allow the practical extension of this work to coherent scattering and speckle analysis.

\section{ACKNOWLEDGEMENTS}

We thank Vincent Cross for fruitful discussions during the preparation of this work. S.F wishes to thank Julián Milano for discussions during the primary phase of development of the algorithm. Financial supports from FLAG-ERA SographMEM (ANR-15-GRFL-0005), funding from the Agence Nationale de la Recherche, France, under grant agreement no. ANR-17-CE240025 (TOPSKY) and 18-CE24-0018-01 (SANTA), the Horizon2020 Framework Program of the European Commission under FET-Proactive Grant agreement no. 824123 (SKYTOP).
[1] J. P. Hannon, G. T. Trammell, M. Blume, and Doon Gibbs. X-ray resonance exchange scattering. Phys. Rev. Lett., 61:1245-1248, Sep 1988.

[2] H. A. Dürr, E. Dudzik, S. S. Dhesi, J. B. Goedkoop, G. van der Laan, M. Belakhovsky, C. Mocuta, A. Marty, and Y. Samson. Chiral magnetic domain structures in ultrathin FePd films. Science, 284(5423):2166-2168, 1999.

[3] G. Beutier, G. van der Laan, K. Chesnel, A. Marty, M. Belakhovsky, S. P. Collins, E. Dudzik, J.-C. Toussaint, and B. Gilles. Characterization of FePd bilayers and trilayers using soft $\mathrm{x}$-ray resonant magnetic scattering and micromagnetic modeling. Phys. Rev. B, 71:184436, May 2005.

[4] Jean-Yves Chauleau, William Legrand, Nicolas Reyren, Davide Maccariello, Sophie Collin, Horia Popescu, Karim Bouzehouane, Vincent Cros, Nicolas Jaouen, and Albert Fert. Chirality in magnetic multilayers probed by the symmetry and the amplitude of dichroism in $\mathrm{x}$ ray resonant magnetic scattering. Phys. Rev. Lett., 120:037202, Jan 2018.

[5] B. Pfau, S. Schaffert, L. Müller, C. Gutt, A. AlShemmary, F. Büttner, R. Delaunay, S. Düsterer, S. Flewett, R. Frömter, J. Geilhufe, E. Guehrs, C. M. Günther, R. Hawaldar, M. Hille, N. Jaouen, A. Kobs,
K. Li, J. Mohanty, H. Redlin, W. F. Schlotter, D. Stickler, R. Treusch, B. Vodungbo, M. Kläui, H. P. Oepen, J. Lüning, G. Grübel, and S. Eisebitt. Ultrafast optical demagnetization manipulates nanoscale spin structure in domain walls. Nature Communications, 3(1):1100, Oct 2012.

[6] Samuel Flewett, Thiago J. A. Mori, Alexandra Ovalle, Simón Oyarzún, Antonio Ibáñez, Sebastián Michea, Juan Escrig, and Juliano Denardin. Soft x-ray magnetic scattering studies of $3 \mathrm{~d}$ magnetic morphology along buried interfaces in $\mathrm{NiFe} / \mathrm{CoPd} / \mathrm{NiFe}$ nanostructures. Scientific Reports, 9(1):14823, Oct 2019.

[7] J.P. Hill and D.F. McMorrow. X-ray resonant exchange scattering: Polarization dependence and correlation functions. Acta Crystallographica. Section A: Foundations of Crystallography, 52:236-244, 1996.

[8] J. M. Tonnerre, L. Sève, D. Raoux, G. Soullié, B. Rodmacq, and P. Wolfers. Soft x-ray resonant magnetic scattering from a magnetically coupled $\mathrm{Ag} / \mathrm{Ni}$ multilayer. Phys. Rev. Lett., 75:740-743, Jul 1995.

[9] J. Zak, E. R. Moog, C. Liu, and S. D. Bader. Fundamental magneto-optics. Journal of Applied Physics, 68(8):4203-4207, 1990.

[10] J. Zak, E. R. Moog, C. Liu, and S. D. Bader. Universal approach to magneto-optics. Journal of Magnetism and 
Magnetic Materials, 89(1-2):107-123, September 1990.

[11] J. Zak, E. R. Moog, C. Liu, and S. D. Bader. Magnetooptics of multilayers with arbitrary magnetization directions. Phys. Rev. B, 43:6423-6429, Mar 1991.

[12] J. Zak, E. R. Moog, C. Liu, and S. D. Bader. Erratum: Magneto-optics of multilayers with arbitrary magnetization directions. Phys. Rev. B, 46:5883-5883, Sep 1992.

[13] M. J. Donahue, D. G. Porter, National Institute of Standards, and Technology (U.S.). OOMMF user's guide /microform/ M.J. Donahue, D.G. Porter. U.S. Dept. of Commerce, Technology Administration, National Institute of Standards and Technology Gaithersburg, MD, version 1.0. edition, 1999.

[14] Arne Vansteenkiste, Jonathan Leliaert, Mykola Dvornik, Mathias Helsen, Felipe Garcia-Sanchez, and Bartel Van Waeyenberge. The design and verification of mumax3. AIP Advances, 4(10):107133, 2014.

[15] J.-Y. Chauleau, T. Chirac, S. Fusil, V. Garcia, W. Akhtar, J. Tranchida, P. Thibaudeau, I. Gross, C. Blouzon, A. Finco, M. Bibes, B. Dkhil, D. D. Khalyavin, P. Manuel, V. Jacques, N. Jaouen, and M. Viret. Electric and antiferromagnetic chiral textures at multiferroic domain walls. Nature Materials, 19(4):386-390, Apr 2020.

[16] S. Fin, R. Tomasello, D. Bisero, M. Marangolo, M. Sacchi, H. Popescu, M. Eddrief, C. Hepburn, G. Finocchio, M. Carpentieri, A. Rettori, M. G. Pini, and $\mathrm{S}$. Tacchi. In-plane rotation of magnetic stripe domains in $\mathrm{Fe}_{1-x} \mathrm{Ga}_{x}$ thin films. Phys. Rev. B, 92:224411, Dec 2015.

[17] William Legrand, Jean-Yves Chauleau, Davide Maccariello, Nicolas Reyren, Sophie Collin, Karim Bouzehouane, Nicolas Jaouen, Vincent Cros, and Albert Fert. Hybrid chiral domain walls and skyrmions in magnetic multilayers. Science Advances, 4(7), 2018.

[18] Shilei Zhang, Gerrit van der Laan, Jan Müller, Lukas Heinen, Markus Garst, Andreas Bauer, Helmuth Berger, Christian Pfleiderer, and Thorsten Hesjedal. Reciprocal space tomography of $3 \mathrm{~d}$ skyrmion lattice order in a chiral magnet. Proceedings of the National Academy of Sciences, 115(25):6386-6391, 2018.

[19] Wenjing Li, Iuliia Bykova, Shilei Zhang, Guoqiang Yu, Riccardo Tomasello, Mario Carpentieri, Yizhou Liu, Yao Guang, Joachim Gräfe, Markus Weigand, David M. Burn, Gerrit van der Laan, Thorsten Hesjedal, Zhengren Yan, Jiafeng Feng, Caihua Wan, Jinwu Wei, Xiao Wang, Xiaomin Zhang, Hongjun Xu, Chenyang Guo, Hongxiang Wei, Giovanni Finocchio, Xiufeng Han, and Gisela Schütz. Anatomy of skyrmionic textures in magnetic multilayers. Advanced Materials, 31(14):1807683, 2019.

[20] S. L. Zhang, G. van der Laan, W. W. Wang, A. A. Haghighirad, and T. Hesjedal. Direct observation of twisted surface skyrmions in bulk crystals. Phys. Rev. Lett., 120:227202, May 2018.

[21] J Díaz, P Gargiani, C Quirós, C Redondo, R Morales, L M Álvarez-Prado, J I Martín, A Scholl, S Ferrer, M Vélez, and S M Valvidares. Chiral asymmetry detected in a $2 \mathrm{~d}$ array of permalloy square nanomagnets using circularly polarized x-ray resonant magnetic scattering. Nanotechnology, 31(2):025702, oct 2019.

[22] E. Burgos-Parra, S.Flewett, Y.Sassi, W.Legrand, F. Ajejas, C. Leveille, Pierluige Gargiani, M. Valdivares, N. Reyren, Vincent Cros, and N.Jaouen. Study of domain walls under in-plane applied magnetic fields using x-ray magnetic resonant scattering. In Preparation.

[23] B. Pianciola, S. Flewett, E. De Biasi, C. Hepburn, L. Lounis, M. Vásquez-Mansilla, M. Granada, M. Barturen, M. Eddrief, M. Sacchi, M. Marangolo, and J. Milano. Magnetoresistance in $\mathrm{Fe}_{0.8} \mathrm{Ga}_{0.2}$ thin films with magnetic stripes: The role of the three-dimensional magnetic structure. Phys. Rev. B, 102:054438, Aug 2020.

[24] C. Léveille, S. Flewett, Y. Sassi, M. Garrido, N. Jaouen, N. Reyren, W. Legrand, F. Ajejas, and V. Cros. Study of domain walls under in-plane applied magnetic fields using x-ray magnetic resonant scattering. In Preparation.

[25] Samuel Flewett, Durgamadhab Mishra, Thiago J. A. Mori, Christian M. Günther, Juliano C. Denardin, Simón Oyarzún, Sebastián Michea, Dieter Engel, Manuel Fohler, Tulio C. R. Rocha, Alexandra Ovalle F., Leandro T. Núñez A., Bastian Pfau, Juan Escrig, and Stefan Eisebitt. Three-dimensional characterization of $\mathrm{Co} / \mathrm{Pd}$ multilayer thin films using resonant soft $\mathrm{x}$-ray scattering. Phys. Rev. B, 95:094430, Mar 2017.

[26] Shilei Zhang, David M. Burn, Nicolas Jaouen, JeanYves Chauleau, Amir A. Haghighirad, Yizhou Liu, Weiwei Wang, Gerrit van der Laan, and Thorsten Hesjedal. Robust perpendicular skyrmions and their surface confinement. Nano Letters, 20(2):1428-1432, Feb 2020.

[27] Roger G. Newton. Optical theorem and beyond. American Journal of Physics, 44(7):639-642, 1976.

[28] Gerrit van der Laan. Soft x-ray resonant magnetic scattering of magnetic nanostructures. Comptes Rendus Physique, 9(5):570 - 584, 2008. Synchrotron x-rays and condensed matter.

[29] S Macke and E Goering. Magnetic reflectometry of heterostructures. Journal of Physics: Condensed Matter, 26(36):363201, aug 2014.

[30] M. Elzo, E. Jal, O. Bunau, S. Grenier, Y. Joly, A.Y. Ramos, H.C.N. Tolentino, J.M. Tonnerre, and N. Jaouen. X-ray resonant magnetic reflectivity of stratified magnetic structures: Eigenwave formalism and application to a $\mathrm{W} / \mathrm{Fe} / \mathrm{W}$ trilayer. Journal of Magnetism and Magnetic Materials, 324(2):105 - 112, 2012.

[31] L. G. Parratt. Surface studies of solids by total reflection of x-rays. Phys. Rev., 95:359-369, Jul 1954.

[32] M. Mansuripur. Analysis of multilayer thin-film structures containing magneto-optic and anisotropic media at oblique incidence using 22 matrices. Journal of Applied Physics, 67(10):6466-6475, 1990.

[33] A. Bourzami, O. Lenoble, Ch. Féry, J. F. Bobo, and M. Piecuch. Enhancement of polar kerr rotation in $\mathrm{Fe} / \mathrm{Al}_{2} \mathrm{O}_{3}$ multilayers and composite systems. Phys. Rev. B, 59:11489-11494, May 1999.

[34] Z. Q. Qiu and S. D. Bader. Surface magneto-optic kerr effect. Review of Scientific Instruments, 71(3):1243-1255, 2000.

[35] J. M. Tonnerre, M. De Santis, S. Grenier, H. C. N. Tolentino, V. Langlais, E. Bontempi, M. GarcíaFernández, and U. Staub. Depth magnetization profile of a perpendicular exchange coupled system by softx-ray resonant magnetic reflectivity. Phys. Rev. Lett., 100:157202, Apr 2008.

[36] J.-M. Tonnerre, E. Jal, E. Bontempi, N. Jaouen, M. Elzo, S. Grenier, H. L. Meyerheim, and M. Przybylski. Depthresolved magnetization distribution in ultra thin films by soft x-ray resonant magnetic reflectivity. The European Physical Journal Special Topics, 208(1):177-187, 2012. 
[37] H.-Ch. Mertins, S. Valencia, D. Abramsohn, A. Gaupp, W. Gudat, and P. M. Oppeneer. X-ray kerr rotation and ellipticity spectra at the $2 p$ edges of Fe, Co, and Ni. Phys. Rev. B, 69:064407, Feb 2004.

[38] T. Kuschel, C. Klewe, J.-M. Schmalhorst, F. Bertram, O. Kuschel, T. Schemme, J. Wollschläger, S. Francoual, J. Strempfer, A. Gupta, M. Meinert, G. Götz, D. Meier, and G. Reiss. Static magnetic proximity effect in $\mathrm{Pt} / \mathrm{NiFe}_{2} \mathrm{O}_{4}$ and $\mathrm{Pt} / \mathrm{B}$ bilayers investigated by $\mathrm{x}-$ ray resonant magnetic reflectivity. Phys. Rev. Lett., 115:097401, Aug 2015.

[39] C. Klewe, T. Kuschel, J.-M. Schmalhorst, F. Bertram, O. Kuschel, J. Wollschläger, J. Strempfer, M. Meinert, and G. Reiss. Static magnetic proximity effect in $\mathrm{Pt} / \mathrm{Ni}_{1-x} \mathrm{Fe}_{x}$ bilayers investigated by x-ray resonant magnetic reflectivity. Phys. Rev. B, 93:214440, Jun 2016.

[40] Sebastian Macke, Abdullah Radi, Jorge E. HamannBorrero, Adriano Verna, Martin Bluschke, Sebastian Brück, Eberhard Goering, Ronny Sutarto, Feizhou He, Georg Cristiani, Meng Wu, Eva Benckiser, Hanns-Ulrich Habermeier, Gennady Logvenov, Nicolas Gauquelin, Gianluigi A. Botton, Adam P. Kajdos, Susanne Stemmer, Georg A. Sawatzky, Maurits W. Haverkort, Bernhard Keimer, and Vladimir Hinkov. Element specific monolayer depth profiling. Advanced Materials, pages 6554-6559, 2014.

[41] M Zwiebler, J E Hamann-Borrero, M Vafaee, P Komissinskiy, S Macke, R Sutarto, F He, B Büchner, G A Sawatzky, L Alff, and J Geck. Electronic depth profiles with atomic layer resolution from resonant soft x-ray reflectivity. New Journal of Physics, 17(8):083046, aug 2015.

[42] S. Valencia, A. Gaupp, W. Gudat, Ll. Abad, Ll. Balcells, and B. Martinez. X-ray magnetic circular dichroism in reflection geometry: A tool for investigating surface magnetism in thin films. Journal of Applied Physics, 104(2):023903, 2008.

[43] F. D'Orazio, F. Giammaria, and F. Lucari. Multiple reflection phenomena in magneto-optical studies of thick samples. Journal of Magnetism and Magnetic Materials, 133(1):419 - 421, 1994.

[44] F D'Orazio, F Giammaria, and F Lucari. Magnetooptical studies of yig(cage) thick film. Journal of Magnetism and Magnetic Materials, 140-144:2123 - 2124, 1995. International Conference on Magnetism.

[45] George H. Vineyard. Grazing-incidence diffraction and the distorted-wave approximation for the study of surfaces. Phys. Rev. B, 26:4146-4159, Oct 1982.

[46] B.L. Henke, E.M. Gullikson, and J.C. Davis. X-ray interactions: Photoabsorption, scattering, transmission, and reflection at $\mathrm{e}=50-30,000 \mathrm{ev}, \mathrm{z}=1-92$. Atomic Data and Nuclear Data Tables, 54(2):181 - 342, 1993.

[47] Ne-Te Duane Loh, Stefan Eisebitt, Samuel Flewett, and Veit Elser. Recovering magnetization distributions from their noisy diffraction data. Phys. Rev. E, 82:061128, Dec 2010.

[48] S. A. Kovalenko. Descartes-snell law of refraction with absorption. Semiconductor Physics, Quantum Electronics Optoelectronics, 4:214-218, 2001.

[49] D. R. Lee, S. K. Sinha, D. Haskel, Y. Choi, J. C. Lang, S. A. Stepanov, and G. Srajer. X-ray resonant magnetic scattering from structurally and magnetically rough interfaces in multilayered systems. i. specular reflectivity. Phys. Rev. B, 68:224409, Dec 2003.
[50] D. R. Lee, S. K. Sinha, C. S. Nelson, J. C. Lang, C. T. Venkataraman, G. Srajer, and R. M. Osgood. Xray resonant magnetic scattering from structurally and magnetically rough interfaces in multilayered systems. ii. diffuse scattering. Phys. Rev. B, 68:224410, Dec 2003.

[51] A. Scherz, W. F. Schlotter, K. Chen, R. Rick, J. Stöhr, J. Lüning, I. McNulty, Ch. Günther, F. Radu, W. Eberhardt, O. Hellwig, and S. Eisebitt. Phase imaging of magnetic nanostructures using resonant soft x-ray holography. Phys. Rev. B, 76:214410, Dec 2007.

[52] S. Eisebitt, J. Lüning, W. F. Schlotter, M. Lörgen, O. Hellwig, W. Eberhardt, and J. Stöhr. Lensless imaging of magnetic nanostructures by x-ray spectro-holography. Nature, 432(7019):885-888, Dec 2004.

[53] Claire Donnelly, Manuel Guizar-Sicairos, Valerio Scagnoli, Sebastian Gliga, Mirko Holler, Jörg Raabe, and Laura J. Heyderman. Three-dimensional magnetization structures revealed with x-ray vector nanotomography. Nature, 547(7663):328-331, Jul 2017.

[54] Peng Li and Andrew Maiden. Multi-slice ptychographic tomography. Scientific Reports, 8(1):2049, Feb 2018.

[55] Samuel Flewett, Harry M. Quiney, Chanh Q. Tran, and Keith A. Nugent. Extracting coherent modes from partially coherent wavefields. Opt. Lett., 34(14):21982200, Jul 2009.

[56] Nicolas Jaouen, Jean-Marc Tonnerre, Grigor Kapoujian, Pierre Taunier, Jean-Paul Roux, Denis Raoux, and Fausto Sirotti. An apparatus for temperature-dependent soft X-ray resonant magnetic scattering. Journal of Synchrotron Radiation, 11(4):353-357, Jul 2004.

[57] M Sacchi, N Jaouen, H Popescu, R Gaudemer, J M Tonnerre, S G Chiuzbaian, C F Hague, A Delmotte, J M Dubuisson, G Cauchon, B Lagarde, and F Polack. The SEXTANTS beamline at SOLEIL: a new facility for elastic, inelastic and coherent scattering of soft x-rays. Journal of Physics: Conference Series, 425(7):072018, mar 2013.

[58] R. Soucaille, M. Belmeguenai, J. Torrejon, J.-V. Kim, T. Devolder, Y. Roussigné, S.-M. Chérif, A. A. Stashkevich, M. Hayashi, and J.-P. Adam. Probing the dzyaloshinskii-moriya interaction in cofeb ultrathin films using domain wall creep and brillouin light spectroscopy. Phys. Rev. B, 94:104431, Sep 2016.

[59] Avinash Kumar Chaurasiya, Chandrima Banerjee, Santanu Pan, Sourav Sahoo, Samiran Choudhury, Jaivardhan Sinha, and Anjan Barman. Direct observation of interfacial dzyaloshinskii-moriya interaction from asymmetric spin-wave propagation in $\mathrm{W} / \mathrm{CoFeB} / \mathrm{SiO}_{2}$ heterostructures down to subnanometer cofeb thickness. Scientific Reports, 6(1):32592, Sep 2016.

[60] Philippa M. Shepley, Harry Tunnicliffe, Kowsar Shahbazi, Gavin Burnell, and Thomas A. Moore. Magnetic properties, domain-wall creep motion, and the dzyaloshinskii-moriya interaction in $\mathrm{Pt} / \mathrm{Co} / \mathrm{Ir}$ thin films. Phys. Rev. B, 97:134417, Apr 2018.

[61] S. McVitie, S. Hughes, K. Fallon, S. McFadzean, D. McGrouther, M. Krajnak, W. Legrand, D. Maccariello, S. Collin, K. Garcia, N. Reyren, V. Cros, A. Fert, K. Zeissler, and C. H. Marrows. A transmission electron microscope study of néel skyrmion magnetic textures in multilayer thin film systems with large interfacial chiral interaction. Scientific Reports, 8(1):5703, Apr 2018. 